\title{
On Sampling and Coding for Distributed Acoustic Sensing
}

\author{
Robert L. Konsbruck, Member, IEEE, Emre Telatar, Fellow, IEEE, and Martin Vetterli, Fellow, IEEE
}

\begin{abstract}
The issue of how to efficiently represent the data collected by a network of microphones recording spatio-temporal acoustic wave fields is addressed. Each sensor node in the network samples the sound field, quantizes the samples and transmits the encoded samples to some central unit, which computes an estimate of the original sound field based on the information received from all the microphones. Our analysis is based on the spectral properties of the sound field, which are induced by the physics of wave propagation and have a significant impact on the efficiency of the chosen sampling lattice and coding scheme. As field acquisition by a sensor network typically implies spatio-temporal sampling of the field, a multidimensional sampling theorem for homogeneous random fields with compactly supported spectral measures is proved. To assess the loss of information implied by source coding, rate distortion functions for various coding schemes and sampling lattices are determined. In particular, centralized coding, independent coding and some multiterminal schemes are compared. Under the assumption of spectral whiteness of the sound field, it is shown that sampling with a quincunx lattice followed by independent coding is optimal as it achieves the lower bound given by centralized coding.
\end{abstract}

Index Terms-Multidimensional sampling, rate distortion functions, sensor networks, sound waves, source coding, wave equation.

\section{INTRODUCTION}

$\mathbf{M}$ OST common applications of sensor networks involve some form of area monitoring. The sensor nodes are deployed over a region in order to monitor the evolution of a physical field such as temperature, pressure or light. They collect samples of the observed field, use their processing abilities to carry out some computations on these samples, and transmit the

Manuscript received July 17, 2010; revised August 14, 2011; accepted November 29, 2011. Date of publication January 31, 2012; date of current version April 17, 2012. This work was supported by the National Center of Competence in Research on Mobile Information and Communication Systems (NCCR-MICS, http://www.mics.org), a center supported by the Swiss National Science Foundation under Grant 5005-67322. The material in this paper was presented in part at the 2006 IEEE International Conference on Acoustics, Speech, and Signal Processing.

R. L. Konsbruck and E. Telatar are with the School of Computer and Communication Sciences (I\&C), École Polytechnique Fédérale de Lausanne (EPFL), CH-1015 Lausanne, Switzerland (e-mail: robert.konsbruck@a3.epfl.ch; emre. telatar@epfl.ch).

M. Vetterli is with the School of Computer and Communication Sciences (I\&C), École Polytechnique Fédérale de Lausanne (EPFL), CH-1015 Lausanne, Switzerland, and also with the Department of Electrical Engineering and Computer Science (EECS), University of California, Berkeley, CA 94720 USA (e-mail: martin.vetterli@epfl.ch).

Communicated by A. Nosratinia, Associate Editor for Communication Networks.

Color versions of one or more of the figures in this paper are available online at http://ieeexplore.ieee.org.

Digital Object Identifier 10.1109/TIT.2012.2184849 partially processed data to a central unit (base station), where the measured field is approximately reconstructed. Since the data collected by the sensor nodes is not generated by independent sources but results from observing a physical phenomenon, it has a particular spatio-temporal structure that is determined by the laws of physics. This structure has a significant impact on many aspects of a wireless sensor network, from the quality of the chosen sampling configuration to the efficiency of the implemented compression and communication schemes. A thorough understanding of the physical phenomenon under observation is crucial for the successful design of sensor network architectures.

Since sensor nodes are typically small and self-powered devices, they have limited resources such as energy, memory, computational speed and bandwidth. Moreover, the energy provisioned for a wireless sensor node is not expected to be renewed throughout its mission. Hence, power consumption is the most critical consideration in a wireless sensor network, directly influencing the network's lifetime. While on-board data processing consumes valuable energy, wireless communication with neighboring nodes and the base station is the dominant factor in a sensor node's energy budget. Therefore, to avoid the transmission of redundant information to the base station, the sensor nodes have to use appropriate data compression schemes to reduce the inherent redundancy of the collected data sets. To further reduce the communication needs, it is essential to have the nodes process the data in a distributed fashion, i.e., without access to the neighboring nodes' measurements, so that only minimal (or no) internode communication is required. In keeping with the digital nature of current off-the-shelf communication and data processing devices, we assume that the physical field monitored by the sensor network has to be transformed into sequences of binary digits, even though it is known that Shannon's (source-channel) separation theorem does not necessarily hold for general multiterminal networks [1]-[3]. As the sensor networks considered in this paper correspond to the "expanding sensor network" model studied in [4] and [5], for which the separation theorem has been shown to hold asymptotically, as the number of nodes grows to infinity, this is a reasonable assumption to make.

In this paper, we study the efficient representation of the observed physical field under the aforementioned constraints, which proves to be one of the key issues in sensor network engineering. We address this issue in the context of acoustic wave field acquisition by means of an array of sensor nodes equipped with microphones. The source coding problem for heat diffusion, i.e., when the physical field under consideration is the temperature, has been studied in [6]. While the present paper's focus lies on acoustic sensing, our results are mainly based on the expression of a solution of the wave equation 
in free field, so that our analysis can be carried over to any physical field that obeys this kind of equation, in particular to electromagnetic waves.

While the recorded acoustic field is continuous in space and time, the sensor nodes observe it only at specific locations, corresponding to their own position, and, at least in the prevailing digital setups, at discrete time instants. Field acquisition by a sensor network thus implies spatio-temporal sampling of the field and raises the question regarding the sufficiency of such a discrete-space and -time representation of an analog field. While for nonrandom, one-dimensional signals, an answer to that question is provided by the classical sampling theorem for bandlimited functions, due to Shannon and Nyquist, the situation is slightly more complicated in sensor networks. Indeed, while sampling in time may be adjusted online by reconfiguring the processing performed by the sensor nodes, sampling in space is determined by the layout of the network at deployment time. Moreover, since sampling is inherently multidimensional in sensor networks, the design of an efficient sampling lattice requires taking advantage of the additional degrees of freedom. We prove a spatio-temporal sampling theorem for homogeneous random fields with compactly supported spectral measures and sampling lattices satisfying a mild regularity condition. Even though analogous results are often accepted without proof in the engineering literature, we are not aware of any previous formal proof. We also explain how the geometrical shape of the random field's spectrum should guide the choice of a particular sampling geometry.

While, under appropriate conditions, a discrete-space and -time representation of the analog field is sufficient, discretizing the field's amplitude, also referred to as source coding, implies an unavoidable loss of information. The source coding problem in sensor networks typically amounts to finding the optimal tradeoff between the quality of the central unit's estimate and the amount of resources used by the sensor nodes. This tradeoff may be expressed in terms of a rate distortion function. We study various source coding schemes, differing by the amount of required internode communication and the complexity of the involved maps. In particular, we compare centralized coding (all the samples are available at a single encoder), independent coding (separate encoding and decoding), and multiterminal coding (distributed encoding, joint decoding). The multiterminal coding scheme is of particular interest in sensor network engineering as it makes use of the spatio-temporal structure of the collected data without requiring internode communication. Under the assumption of spectral whiteness of the sound field 1, which essentially corresponds to the worst-case data source in terms of source coding performance, we show that sampling with a quincunx lattice followed by independent coding achieves the lower bound given by centralized coding, thus establishing the multiterminal rate distortion function for the particular setup considered in the paper.

The main objective that we pursue in this paper consists in devising a holistic model incorporating physics, multidimensional sampling and rate distortion theory to evaluate the source coding performance of sensor networks in environmental mon-

\footnotetext{
${ }^{1}$ The spectral density of the recorded sound field is assumed to be constant on its support, which requires a particular correlation structure of the source field.
}

itoring applications. Since the correlation properties of the data collected by the sensor nodes are determined by the laws of physics, the reliable assessment of the minimal information rate needed to accurately represent the recorded samples requires a thorough understanding of the physical phenomenon monitored by the sensor nodes. Therefore, we devote the first part of our work to a rigorous derivation of a solution of the wave equation driven by a bandlimited, Gaussian random field. The approximately bow-tie-shaped support of the recorded field's spectral measure proves indeed to be one of the key ingredients in the proof of our paper's main result. Once the problem is set on a solid basis, we investigate the interplay between multidimensional sampling and source coding in the context of physical fields. Indeed, rather than in designing novel sampling schemes or in establishing new, purely information-theoretic results, our paper's main ambition consists in illustrating the potential gains that may be achieved by a judicious adjustment of the sampling geometry and the source coding scheme to the physical properties of the data sources monitored by the sensor network. In particular, for the worst-case data source, we show that traditional point-to-point source coding leads to the optimal rate distortion tradeoff (the one achieved by multiterminal source coding), as long as it is preceded by sampling with the appropriate quincunx lattice. In other words, the dexterous choice of the sampling geometry exempts us from having to resort to any multiterminal binning, which would be computationally far more intensive than point-to-point coding.

While it stands to reason that a comprehensive analysis of sensor networks requires to take the physics of the underlying phenomenon into account and to address the issues of sampling and source coding jointly, previous work has rarely adopted such a holistic approach to explore the connections among these three fields. For instance, the influence of the spectral measure's decay in the wavenumber-frequency plane on the accuracy of the interpolated sound field has been studied in [7], but the analysis is limited to deterministic fields and to signal-to-noise ratio estimations. Our paper supplements [7] with a random field model and an information-theoretic performance analysis. Another subfield of information technology that physics has recently found its way into is multiple-antenna communication. Indeed, whereas our study is sort of set at the output of the physical channel insofar as we address the issue of efficiently representing the sound field once it has passed through the propagation medium, the problem of devising realistic models for the physical channel itself, with the aim of estimating the capacity scaling in wireless communication networks, has gained a lot of attention over the last decade. Early results have suggested that, under the assumption of a channel model characterized by independent and identically distributed fading across all antenna pairs, the capacity of a multiple-antenna system grows proportionally with the minimum of the numbers of transmitters and receivers [8], [9]. By resorting to physical models of multiple-antenna channels, recent work has shown, however, that the capacity scaling of wireless networks is subject to a fundamental limitation that is due to the laws of physics [10]-[14]. Although these papers focus on monochromatic, electromagnetic signals for communication purposes, they suggest that, in general, the number of degrees of freedom per unit length 
in linear antenna arrays with full angular spread is limited to $\Omega_{0} /(\pi c)$, where $\Omega_{0}$ represents the carrier frequency and $c$ denotes the propagation speed [11, Sect. 7.3]. In the present paper, we show the relevance of this upper bound for the problem of spatio-temporal sampling of sound fields. Indeed, we argue that the wavenumber bandwidth of a sound field with frequency bandwidth $\Omega_{0}$ is upper bounded by $\Omega_{0} / c$, so that $\Omega_{0} /(\pi c)$ spatial samples per unit length are sufficient to accurately represent this sound field. Hence, whereas this physical constraint on the number of degrees of freedom limits the communication performance of multiple-antenna systems, it enhances the source coding performance of sensor networks by reducing the required number of spatial measurements.

The paper is organized as follows. In Section II, we study the equation of sound propagation and derive its solution for the linear sensor array configuration. Then, in Section III, we provide a stochastic model for the source term in the wave equation. Periodic sampling of the acoustic field with different geometries is studied in Section IV, while in Section V, we specify the source coding schemes that we let the sensor nodes apply to the sampled field. Finally, in Section VI, we determine the rate distortion functions for the various sampling lattices and coding schemes.

\section{Sound Waves And the Wave Equation}

In this section, we introduce the common physical model for sound propagation, starting with a generic setup and then specializing the formulas to the linear array configuration, which is the model that we adhere to throughout the rest of the paper. We study the spectral characteristics of the sound field and explain how to determine a solution of the wave equation when the source is a homogeneous, Gaussian random field. The restriction of our study to a shift-invariant setup is entailed by the fact that the calculation of rate distortion functions for general data sources with memory is notoriously difficult $[15$, Sect. 4.4]. To date, single-letter rate distortion functions are known almost exclusively in situations involving stationary (and mostly Gaussian) sources. This explains why we restrict our attention to homogeneous random fields, which allows us to properly define spectral densities and to infer the various rate distortion functions from the Toeplitz distribution theorem. Since the domain of definition of homogeneous fields has to exhibit sufficient symmetry, we have chosen to focus our work on setups based on linear arrangements of the sound sources and the microphones.

\section{A. The Wave Equation in Free Field}

We restrict our attention to wave motion in an ideal, homogeneous, compressible and nonviscous fluid, which is at rest in thermodynamic equilibrium, except for the motion caused by the sound waves themselves. Furthermore, we assume that this acoustic motion is sufficiently small in magnitude so that any nonlinear effects are negligible, and that the compression of the fluid is adiabatic. The fluid is characterized by its adiabatic compressibility $\kappa$, its equilibrium density $\rho$ and its equilibrium pressure $V_{0}$. Let $V(\boldsymbol{x}, t)$, for $\boldsymbol{x}=\left(x_{1}, x_{2}, x_{3}\right) \in \mathbb{R}^{3}$ and $t \in \mathbb{R}_{+}=[0,+\infty)$, denote the acoustic pressure at position $\boldsymbol{x}$ and at time $t$, i.e., the pressure change caused by the sound at position $x$ and at time $t$. Let $U: \mathbb{R}^{3} \times \mathbb{R}_{+} \rightarrow \mathbb{R}$ denote an external sound source such as a loudspeaker. In the formu- lation that we have chosen, $U$ corresponds to $-\nabla \cdot f$, where $f: \mathbb{R}^{3} \times \mathbb{R}_{+} \rightarrow \mathbb{R}^{3}$ denotes the external force density acting on the fluid, and $\nabla \cdot$ denotes the divergence operator with respect to the space variables. The acoustic pressure $V: \mathbb{R}^{3} \times \mathbb{R}_{+} \rightarrow \mathbb{R}$, which we also call the sound field, satisfies the following linear hyperbolic partial differential equation (PDE) [16, Sect. 6.2]:

$\frac{1}{c^{2}} \frac{\partial^{2}}{\partial t^{2}} V(\boldsymbol{x}, t)=\Delta V(\boldsymbol{x}, t)+U(\boldsymbol{x}, t), \quad(\boldsymbol{x}, t) \in \mathbb{R}^{3} \times(0,+\infty)$

where $\Delta:=\sum_{j=1}^{3} \partial^{2} / \partial x_{j}^{2}$ denotes the Laplacian operator with respect to the space variables, and $c:=1 / \sqrt{\kappa \rho}$ is the speed of sound. A function $V: \mathbb{R}^{3} \times \mathbb{R}_{+} \rightarrow \mathbb{R}$ is said to be a solution of (1) if $V$ is an element of $C^{2}\left(\mathbb{R}^{3} \times \mathbb{R}_{+}\right)$, and its partial derivatives $\partial^{2} V / \partial t^{2}$ and $\partial^{2} V / \partial x_{j}^{2}$, for $j=1,2,3$, satisfy (1). To ensure the uniqueness of the solution, we specify initial conditions for $V(\boldsymbol{x}, t)$ and $\partial V(\boldsymbol{x}, t) / \partial t$ at $t=0$. Since we are primarily interested in the effect of the external sound source, we assume that these initial conditions are identically zero. That is, we search for a function $V \in C^{2}\left(\mathbb{R}^{3} \times \mathbb{R}_{+}\right)$solving the following Cauchy problem:

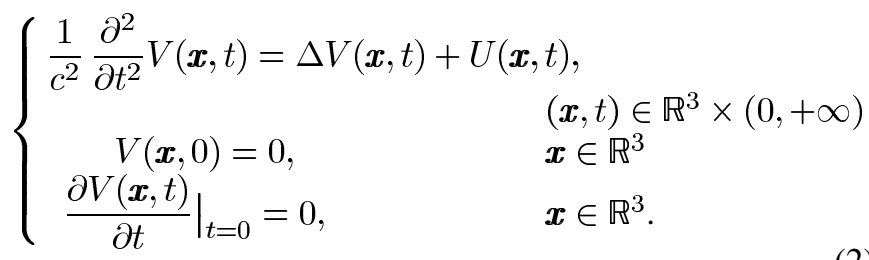

If the function $U$ is an element of $C^{2}\left(\mathbb{R}^{3} \times \mathbb{R}_{+}\right)$, the unique solution of (2) is given by [17, Sect. VI.8]

$$
V(\boldsymbol{x}, t)=\frac{1}{4 \pi} \int_{0}^{t} \frac{1}{\tau} \int_{S^{2}(c \tau)} U(\boldsymbol{x}-\boldsymbol{\xi}, t-\tau) \mathrm{d} \sigma_{c \tau}(\boldsymbol{\xi}) \mathrm{d} \tau
$$

where $S^{2}(\tau):=\left\{\boldsymbol{\xi} \in \mathbb{R}^{3}:\|\boldsymbol{\xi}\|=\tau\right\}$ is the sphere in $\mathbb{R}^{3}$ centered at the origin and of radius $\tau(\|$.$\| denotes the Euclidean$ norm in $\left.\mathbb{R}^{3}\right)$, and $\sigma_{\tau}$ denotes the surface measure on $S^{2}(\tau)$. Performing the change of variable $r:=c \tau$ and combining the iterated integrals into a single one, we rewrite (3) as

$$
\begin{aligned}
V(\boldsymbol{x}, t) & =\frac{1}{4 \pi} \int_{0}^{c t} \int_{S^{2}(r)} \frac{U(\boldsymbol{x}-\boldsymbol{\xi}, t-r / c)}{r} \mathrm{~d} \sigma_{r}(\boldsymbol{\xi}) \mathrm{d} r \\
& =\frac{1}{4 \pi} \int_{B^{3}(c t)} \frac{U(\boldsymbol{x}-\boldsymbol{\xi}, t-\|\boldsymbol{\xi}\| / c)}{\|\boldsymbol{\xi}\|} \mathrm{d} \boldsymbol{\xi}
\end{aligned}
$$

where $B^{3}(\tau):=\left\{\boldsymbol{\xi} \in \mathbb{R}^{3}:\|\boldsymbol{\xi}\|<\tau\right\}$ is the open ball in $\mathbb{R}^{3}$ centered at the origin and of radius $\tau$. Defining the generalized function

$$
g(\boldsymbol{x}, t):=\frac{\delta(t-\|x\| / c)}{4 \pi\|\boldsymbol{x}\|}
$$

where $\delta:=\delta_{0}$ is the Dirac distribution at the origin, we rewrite (4) as

$$
V(\boldsymbol{x}, t)=\int_{\mathbb{R}^{3}} \int_{-\infty}^{t} g(\boldsymbol{\xi}, \tau) U(\boldsymbol{x}-\boldsymbol{\xi}, t-\tau) \mathrm{d} \tau \mathrm{d} \boldsymbol{\xi} .
$$

The function $g$ is called the fundamental solution, or the Green's function, of the wave equation (1). It is the solution, in the distributional sense, of the PDE (1) when the source term $U(x, t)$ is the Dirac distribution at the origin. The function $g$ has also been named the plenacoustic function in [7], [18]. 


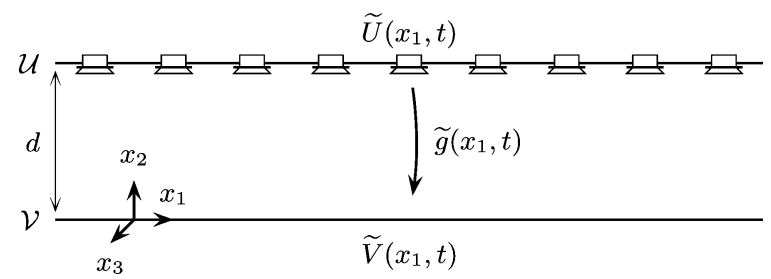

Fig. 1. Setup for studying the sound propagation between two parallel lines. Sound sources concentrated on an infinite line $\mathcal{U}$ emit an acoustic field $\widetilde{U}\left(x_{1}, t\right)$, which induces a sound field $\widetilde{V}\left(x_{1}, t\right)$ on another infinite line $\mathcal{V}$, which is parallel to and at distance $d$ from the line $\mathcal{U}$.

The right-hand side of (4) is well defined under weaker conditions than $U \in C^{2}\left(\mathbb{R}^{3} \times \mathbb{R}_{+}\right)$. However, in such a case, the corresponding function $V$ defined by (4) need not satisfy the PDE (1) in the classical sense. For this reason, we call (4), whenever it is well defined, a weak solution of the Cauchy problem (2). Moreover, we usually model the sound source $U$ as a homogeneous random field defined on $\mathbb{R}^{3} \times \mathbb{R}$. In order for the solution $V$ to be a homogeneous random field as well, its domain of definition must be extended to $\mathbb{R}^{3} \times \mathbb{R}$. We therefore let $V$ be the solution of the Cauchy problem obtained from (2) by replacing $(0,+\infty)$ by $\mathbb{R}$, and the initial conditions by $\lim _{t \rightarrow-\infty} V(\boldsymbol{x}, t)=0, \boldsymbol{x} \in \mathbb{R}^{3}$, and $\lim _{t \rightarrow-\infty} \partial V(\boldsymbol{x}, t) / \partial t=0, \boldsymbol{x} \in \mathbb{R}^{3}$, respectively. Analogously to (4), we define a weak solution of this modified Cauchy problem by

$$
V(\boldsymbol{x}, t):=\frac{1}{4 \pi} \int_{\mathbb{R}^{3}} \frac{U(\boldsymbol{x}-\boldsymbol{\xi}, t-\|\boldsymbol{\xi}\| / c)}{\|\boldsymbol{\xi}\|} \mathrm{d} \boldsymbol{\xi}
$$

which is well defined under appropriate regularity conditions on the function $U$.

\section{B. Linear Array Configuration}

Let us now turn our attention to the setup where the sound field $V$ is to be recorded along a straight line $\mathcal{V}$, which we assume to extend to infinity to be able to work with homogeneous random fields. We further assume that the source field $U$ is emitted from a line $\mathcal{U}$ that is parallel to and at distance $d$ from the line $\mathcal{V}$. This particular choice does not narrow the applicability of our results because, according to Huygens's principle [19], every point of a wave front may be considered as a secondary source, and we can compute the acoustic pressure at a given point by considering the cumulative effect of all these secondary sources. If the sound source generating the wave front, i.e., the primary source, is sufficiently far away from the recording devices, the curvature of the wave front may be neglected so that the wave front may indeed be approximated by a straight line. This principle is at the basis of wave field synthesis [20]. Without loss of generality we assume that both the lines $\mathcal{U}$ and $\mathcal{V}$ lie in the $\left(x_{1}, x_{2}\right)$-plane in $\mathbb{R}^{3}$, and that the line $\mathcal{V}$ coincides with the $x_{1}$ axis, as shown in Fig. 1. The source function $U$ may then be written as

$$
U(\boldsymbol{x}, t):=\widetilde{U}\left(x_{1}, t\right) \delta_{\mathbf{0}}\left(x_{2}-d, x_{3}\right)
$$

for some function $\widetilde{U}: \mathbb{R}^{2} \rightarrow \mathbb{R}$, while the sound field observed along the line $\mathcal{V}$ is given by

$$
\widetilde{V}\left(x_{1}, t\right):=V\left(\left(x_{1}, 0,0\right), t\right) .
$$

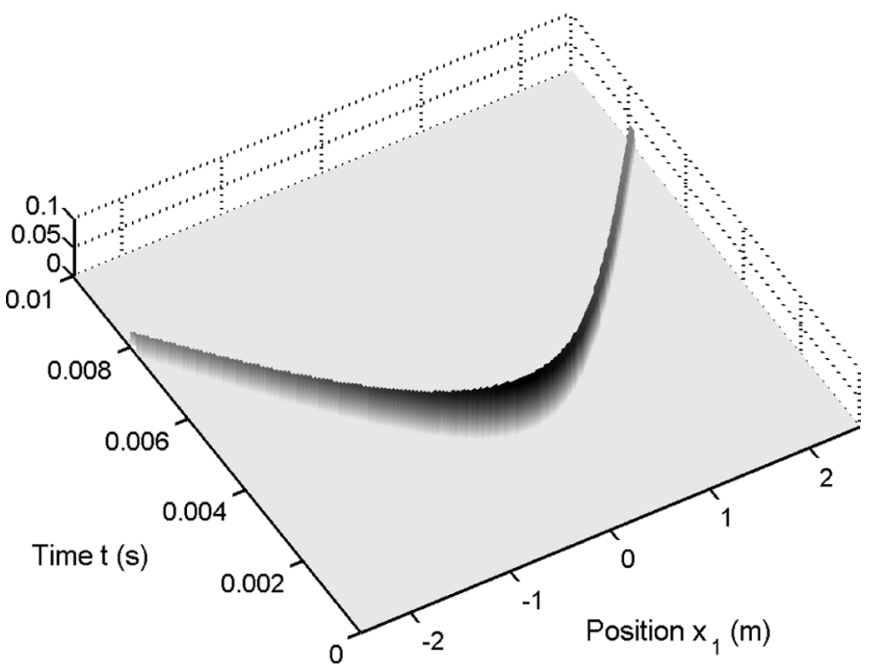

Fig. 2. Green's function for the sound propagation between two parallel lines. It represents the sound field along the line $\mathcal{V}$ induced by a point source on the line $\mathcal{U}$ that generates a unit impulse at position $x_{1}=0$ and at time $t=0$.

It follows from (5) that the functions $\widetilde{U}$ and $\widetilde{V}$ are related by

$$
\widetilde{V}\left(x_{1}, t\right)=\frac{1}{4 \pi} \int_{\mathbb{R}} \frac{\tilde{U}\left(x_{1}-\xi_{1}, t-\sqrt{d^{2}+\xi_{1}^{2}} / c\right)}{\sqrt{d^{2}+\xi_{1}^{2}}} \mathrm{~d} \xi_{1} .
$$

Defining the generalized function

$$
\widetilde{g}\left(x_{1}, t\right):=\frac{\delta\left(t-\sqrt{d^{2}+x_{1}^{2}} / c\right)}{4 \pi \sqrt{d^{2}+x_{1}^{2}}}
$$

we rewrite (6) as

$$
\widetilde{V}\left(x_{1}, t\right)=\iint_{\mathbb{R}^{2}} \widetilde{g}\left(\xi_{1}, \tau\right) \widetilde{U}\left(x_{1}-\xi_{1}, t-\tau\right) \mathrm{d} \tau \mathrm{d} \xi_{1} .
$$

Hence, the function $\widetilde{g}$ may be regarded as the fundamental solution for the sound propagation between the two parallel lines. It is illustrated in Fig. 2 and represents the sound field along the line $\mathcal{V}$ induced by a point source on the line $\mathcal{U}$ that generates a unit impulse at position $x_{1}=0$ and at time $t=0$. Let $\widehat{g}$ denote the Fourier transform of $\widetilde{g}$ in the distributional sense. Then one can show that $[7$, Appendix I]

$$
\widehat{g}\left(\Phi_{1}, \Omega\right)=\left\{\begin{array}{cl}
-\frac{j}{4} H_{0}^{*}\left(d \sqrt{(\Omega / c)^{2}-\Phi_{1}^{2}}\right) & \text { if } \Omega \geq 0 \\
\frac{j}{4} H_{0}\left(d \sqrt{(\Omega / c)^{2}-\Phi_{1}^{2}}\right) & \text { if } \Omega<0
\end{array}\right.
$$

where $\Phi_{1}$ and $\Omega$ represent the wavenumber (spatial frequency) and the frequency (temporal frequency) ${ }^{2}$, respectively, $H_{0}$ denotes the Hankel function of the first kind of order zero, and $H_{0}^{*}$ is the complex conjugate of $H_{0}$. For later reference, we state the following results about the asymptotic forms of the Hankel function [21, Sect. 8.40 and 8.45]:

$$
\left|H_{0}(\nu)\right|^{2}=1+\left(\frac{2}{\pi} \log (\gamma \nu / 2)\right)^{2}+o(\nu) \text { as } \nu \rightarrow 0
$$

${ }^{2}$ We use the following convention for defining the Fourier transform $\widehat{f}$ of an integrable function $f: \mathbb{R}^{2} \rightarrow \mathbb{R}$ :

$$
\widehat{f}\left(\Phi_{1}, \Omega\right)=\iint_{\mathbb{R}^{2}} f\left(x_{1}, t\right) e^{-j\left(\Phi_{1} x_{1}+\Omega t\right)} \mathrm{d} x_{1} \mathrm{~d} t .
$$




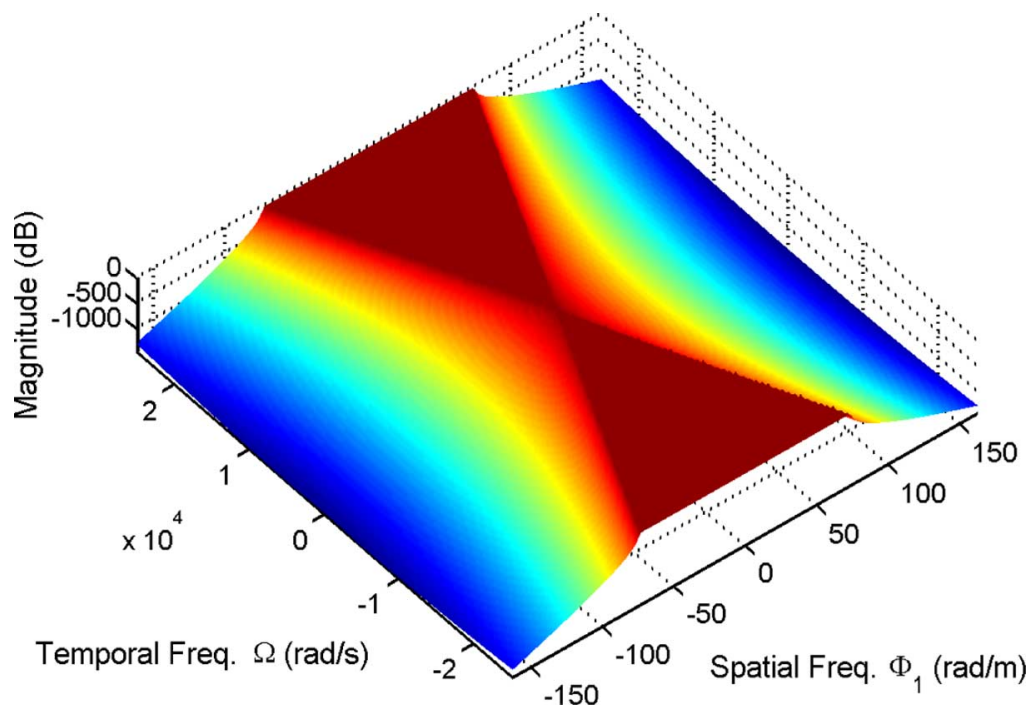

Fig. 3. Magnitude of the fundamental solution's Fourier transform in dB.

$$
\begin{aligned}
\left|H_{0}(\nu)\right|^{2}=\frac{2}{\pi \nu}+o(1 / \nu) & \text { as } \nu \rightarrow \infty \\
\left|H_{0}(j \nu)\right|^{2}=\frac{2}{\pi \nu} e^{-2 \nu}+o\left(e^{-2 \nu} / \nu\right) & \text { as } \nu \rightarrow \infty
\end{aligned}
$$

where $\gamma=e^{C}$, and $C \simeq 0.577$ denotes Euler's constant. The magnitude of $\widehat{g}$ in $\mathrm{dB}$ is illustrated in Fig. 3. Interpreting $\widehat{g}$ as the transfer function of a linear and shift-invariant filter, we note that its passband approximately coincides with the bow-tie shaped region of the wavenumber-frequency pairs $\left(\Phi_{1}, \Omega\right)$ satisfying $\left|\Phi_{1}\right|<|\Omega| / c$. Outside this region the argument of the Hankel function in (8) is purely imaginary, and it follows from (11) that, for fixed $\Omega,\left|\widehat{g}\left(\Phi_{1}, \Omega\right)\right|$ decays exponentially fast as $\left|\Phi_{1}\right| \rightarrow$ $\infty$. We also notice that this exponential decay becomes faster with increasing $d$. For this reason, when the two parallel lines are separated by a sufficiently large distance, we may and will assume that $\widehat{g}\left(\Phi_{1}, \Omega\right)$ vanishes whenever $\left|\Phi_{1}\right|>|\Omega| / c$. This is the so-called far-field approximation of the sound field [7], as opposed to the near-field setting where we consider $\widehat{g}$ to be given by (8). The bow-tie shaped support of the Fourier transform $\hat{g}$ in the far-field approximation is a consequence of ignoring the curvature of the acoustic wave fronts and the attenuation along the line of observation.

To simplify the notation in what follows, we drop the tildes on $\widetilde{U}, \widetilde{V}$, and $\widetilde{g}$, and we relabel $x_{1}, \xi_{1}$, and $\Phi_{1}$ by $x, \xi$, and $\Phi$, respectively.

\section{Stochastic SOURCE Model}

In this section, we describe the stochastic model that we use for the sound source $U$, as well as its implications on the nature of the induced acoustic field $V$. As is usual in engineering modeling, we assume that the source is a real-valued, zero-mean, homogeneous, Gaussian random field $\left\{U(x, t):(x, t) \in \mathbb{R}^{2}\right\}$ defined on a probability space $(\Omega, \mathcal{F}, P)$. We further assume that the field's covariance function $K_{U}: \mathbb{R}^{2} \rightarrow \mathbb{R}$ is continuous, and that the field admits a spectral density $S_{U}: \mathbb{R}^{2} \rightarrow[0,+\infty)$ such that

$$
K_{U}(\xi, \tau)=\frac{1}{(2 \pi)^{2}} \iint_{\mathbb{R}^{2}} S_{U}(\Phi, \Omega) e^{j(\xi \Phi+\tau \Omega)} \mathrm{d} \Phi \mathrm{d} \Omega
$$

In fact, it follows from Kolmogorov's existence theorem [22, Th. 36.1] that for every choice of a nonnegative and Lebesgue integrable function $S_{U}: \mathbb{R}^{2} \rightarrow[0,+\infty)$, there exists a Gaussian random field $\left\{U(x, t):(x, t) \in \mathbb{R}^{2}\right\}$ with spectral density $S_{U}$. Furthermore, the random field can be chosen to be separable and measurable [23, Th. II.2.4 and Th. II.2.6]. That is, the field $U$ is determined, with probability one, by its values taken on a countable dense subset $\mathcal{S} \subset \mathbb{R}^{2}$, and it is measurable as a function of $(x, t, \omega) \in \mathbb{R}^{2} \times \Omega$. Throughout the paper we restrict our attention to source fields $\left\{U(x, t):(x, t) \in \mathbb{R}^{2}\right\}$ whose spectral densities $S_{U}$ are bounded and have compact supports. To be explicit, we assume that the source field is bandlimited to the wavenumber ${ }^{3} \widetilde{\Phi}_{0}$ and to the frequency $\Omega_{0}$, i.e., that its spectral density $S_{U}$ vanishes outside the rectangle $\left(-\widetilde{\Phi}_{0}, \widetilde{\Phi}_{0}\right) \times$ $\left(-\Omega_{0}, \Omega_{0}\right)$.

Analogously to (6), we define a random field $\{V(x, t)$ : $\left.(x, t) \in \mathbb{R}^{2}\right\}$ by

$$
V(x, t):=\frac{1}{4 \pi} \int_{\mathbb{R}} \frac{U\left(x-\xi, t-\sqrt{d^{2}+\xi^{2}} / c\right)}{\sqrt{d^{2}+\xi^{2}}} \mathrm{~d} \xi
$$

In [24, Appendix B], we show that, for each $(x, t) \in \mathbb{R}^{2}$, the integral in (13) exists as an improper Lebesgue integral in the quadratic-mean (q.m.) sense, and $V(x, t)$ is a well-defined

${ }^{3}$ We denote the wavenumber bandwidth of the source by $\widetilde{\Phi}_{0}$ because, unlike its frequency bandwidth $\Omega_{0}$, which always determines the frequency bandwidth of the induced acoustic field, it does not necessarily coincide with the wavenumber bandwidth, denoted by $\Phi_{0}$, of the recorded sound field but only constitutes an upper bound, as we will show later. 
random variable. More explicitly, we prove that, for every $(x, t) \in \mathbb{R}^{2}$, there exists a random variable $V(x, t)$ such that

$$
\begin{array}{r}
\lim _{\substack{\alpha \rightarrow-\infty \\
\beta \rightarrow+\infty}} \mathrm{E}\left[\mid \frac{1}{4 \pi} \int_{\alpha}^{\beta} \frac{U\left(x-\xi, t-\sqrt{d^{2}+\xi^{2}} / c\right)}{\sqrt{d^{2}+\xi^{2}}} \mathrm{~d} \xi\right. \\
\left.-\left.V(x, t)\right|^{2}\right]=0
\end{array}
$$

by arguing that the integral in (14), considered as a function of its integration limits, satisfies, as $\alpha \rightarrow-\infty$ and $\beta \rightarrow+\infty$, a "Cauchy-like" criterion in the Hilbert space $L^{2}(\Omega, \mathcal{F}, P)$ of square-integrable random variables. The only hypotheses that our proof relies on are that the spectral density $S_{U}$ of the source field is bounded and has a compact support. Neither of these assumptions restricts the applicability of our results since the boundedness of $S_{U}$ follows from Bochner's theorem [23, Th. XI.3.2], if we make the reasonable assumption that the source field's covariance function $K_{U}$ is continuous, and the compactness of the support may be assumed without loss of generality, as we argue at the end of the section. Since we are primarily interested in the mean-squared-error fidelity criterion, the interpretation of the integral in (13) as a q.m. linear operation is adequate for our purposes. It follows that $\left\{V(x, t):(x, t) \in \mathbb{R}^{2}\right\}$ is a zero-mean, homogeneous, Gaussian random field, whose spectral density is given by [25, Sect. 3.6]

$$
S_{V}(\Phi, \Omega)=|\widehat{g}(\Phi, \Omega)|^{2} S_{U}(\Phi, \Omega)
$$

where $\widehat{g}$ denotes the Fourier transform of the fundamental solution and is given by (8). As argued in Section II, the field $V$ may be considered to be a weak solution of the sound propagation problem.

While being amenable to the tools of information theory, the source model specified above is sufficiently general to apply to many physical phenomena of interest. Indeed, whenever the observed field is generated by a large number of small effects that act additively and independently, the central limit theorem suggests that the outcome has an approximately Gaussian distribution. Moreover, from a source coding perspective, Gaussian random processes are useful for gaining insight into the worstcase performance of source coding schemes as these processes are the hardest to describe among all random processes with a given covariance function [15, Th. 4.6.3]. As to the assumption of finite bandwidths, most physical fields encountered in practice, even though they are not perfectly bandlimited and no spatial low-pass prefiltering can be applied to these fields in sensor networks, typically contain a large fraction of their total power in a confined spectral domain. To keep the analysis sufficiently simple, we have therefore chosen to restrict our attention to bandlimited fields and not to address the issue of aliasing in our paper. Indeed, we aim at evaluating the net performance of source coding schemes, and aliasing contributes to the overall distortion an additional term that is difficult to evaluate [26]. In Section IV, we argue that, for any bandlimited random field, we can choose a sufficiently dense sampling lattice to enable alias-free interpolation of the field's samples.

\section{SENSOR ARRAY AND SPATIO-TEMPORAL SAMPLING}

As mentioned in the introduction, the acquisition of the sound field $V$ is performed by means of an array of microphones that are deployed on the observation line $\mathcal{V}$. We restrict our attention to periodic sampling and study the representation of the analog field $\left\{V(x, t):(x, t) \in \mathbb{R}^{2}\right\}$ by its samples taken at the vertices of a regular lattice in the spatio-temporal plane. In this section, we look at the relationship between the continuous-parameter field and the sampled field obtained from it by periodic sampling, we illustrate the potential gains in efficiency that can be achieved by adjusting the sampling geometry to the analog field's spectral support, and we address the question of how to reconstruct the analog field from its samples. Note however that only the sampling in the temporal dimension may be adjusted online by reconfiguring the processing performed by the sensors, while the sampling in the spatial dimension is determined by the sensors' locations (unless the sensors are able to move, which we do not assume). In this section, we study two different sampling geometries, obtained from the rectangular and the quincunx lattices, respectively. The issue of periodic sampling with arbitrary sampling geometries has been addressed in [27, Sect. 1.4] for the case of deterministic fields. We generalize the arguments in [27] to random fields and provide a formal proof of the convergence of the interpolating series.

\section{A. Two-Dimensional Periodic Sampling}

For notational convenience we let $z$ denote the vector $(x, t) \in$ $\mathbb{R}^{2}$. Assume that $\left\{V(\boldsymbol{z}): \boldsymbol{z} \in \mathbb{R}^{2}\right\}$ is a bandlimited, Gaussian random field, like the one defined in Section III. A regular lattice in $\mathbb{R}^{2}$ is characterized by a nonsingular $2 \times 2$ matrix $\boldsymbol{M}$ with real coefficients, which we call the sampling matrix (also called the generating matrix in the literature). The points in the set $\Lambda_{\boldsymbol{M}}:=\left\{\boldsymbol{M l}: \boldsymbol{l} \in \mathbb{Z}^{2}\right\}$ are the vertices of the lattice. Sampling the analog field $V$ at these vertices produces the discrete-parameter field $\left\{\widetilde{V}[\boldsymbol{l}]:=V(\boldsymbol{M l}): \boldsymbol{l} \in \mathbb{Z}^{2}\right\}$. The average number of samples per unit area is given by $1 /|\operatorname{det} \boldsymbol{M}|$. Note that the field $\left\{\widetilde{V}[l]: l \in \mathbb{Z}^{2}\right\}$ is a discrete-parameter, zero-mean, homogeneous, Gaussian random field whose covariance function $K_{\widetilde{V}}: \mathbb{Z}^{2} \rightarrow \mathbb{R}$ is given by

$$
\begin{aligned}
K_{\widetilde{V}}[\lambda] & :=\mathrm{E}[\tilde{V}[\boldsymbol{l}+\boldsymbol{\lambda}] \tilde{V}[\boldsymbol{l}]] \\
& =K_{V}(\boldsymbol{M} \boldsymbol{\lambda})
\end{aligned}
$$

where $K_{V}: \mathbb{R}^{2} \rightarrow \mathbb{R}$ denotes the covariance function of the field $\left\{V(\boldsymbol{z}): z \in \mathbb{R}^{2}\right\}$. Expressing $K_{V}$ in terms of the spectral density $S_{V}$, analogously to (12), letting $\Psi$ denote the vector $(\Phi, \Omega) \in \mathbb{R}^{2}$, and performing the change of variable $\boldsymbol{\psi}:=$ $\boldsymbol{M}^{\mathrm{T}} \boldsymbol{\Psi}$, we obtain

$$
\begin{aligned}
K_{\widetilde{V}}[\boldsymbol{\lambda}] & =\frac{1}{(2 \pi)^{2}} \int_{\mathbb{R}^{2}} S_{V}(\boldsymbol{\Psi}) e^{j\langle\boldsymbol{M} \boldsymbol{\lambda}, \boldsymbol{\Psi}\rangle} \mathrm{d} \boldsymbol{\Psi} \\
& =\frac{1}{(2 \pi)^{2}} \int_{\mathbb{R}^{2}} \frac{1}{|\operatorname{det} \boldsymbol{M}|} S_{V}\left(\boldsymbol{M}^{-\mathrm{T}} \boldsymbol{\psi}\right) e^{j\langle\boldsymbol{\lambda}, \boldsymbol{\psi}\rangle} \mathrm{d} \boldsymbol{\psi}
\end{aligned}
$$

where $\langle\cdot, \cdot\rangle$ denotes the canonical inner product of $\mathbb{R}^{2}$, and $M^{-\mathrm{T}}$ is the transposed inverse of the matrix $M$. Splitting the integral over $\mathbb{R}^{2}$ in (16) into an infinite series of integrals, each of 
which is over a square obtained from $(-\pi, \pi]^{2}$ by translation, we rewrite (16) as

$$
\begin{aligned}
K_{\widetilde{V}}[\boldsymbol{\lambda}]= & \frac{1}{(2 \pi)^{2}} \sum_{\boldsymbol{m} \in \mathbb{Z}^{2}} \int_{(-\pi, \pi]^{2}} \frac{1}{|\operatorname{det} \boldsymbol{M}|} \\
= & \frac{1}{(2 \pi)^{2}} \int_{(-\pi, \pi]^{2}} \frac{1}{|\operatorname{det} \boldsymbol{M}|} \\
& \left.\left.\cdot \sum_{\boldsymbol{m} \in \mathbb{Z}^{2}} S_{V}\left(\boldsymbol{M}^{-\mathrm{T}}(\boldsymbol{\psi}-2 \pi \boldsymbol{m})\right) e^{j\langle\boldsymbol{\lambda}, \boldsymbol{\psi}\rangle} \mathrm{d} \boldsymbol{\psi}-2 \pi \boldsymbol{m}\right)\right) e^{j\langle\boldsymbol{\lambda}, \boldsymbol{\psi}\rangle} \mathrm{d} \boldsymbol{\psi}
\end{aligned}
$$

where the interchange of summation and integration is justified by the fact that the series in (17) is a finite sum due to the compact support of $S_{V}$. It follows from (17) and the spectral representation theorem for the covariance functions of discrete-parameter, homogeneous random fields [23, Th. X.3.2] that the spectral density $S_{\widetilde{V}}:(-\pi, \pi]^{2} \rightarrow[0,+\infty)$ of the field $\left\{\tilde{V}[\boldsymbol{l}]: \boldsymbol{l} \in \mathbb{Z}^{2}\right\}$ is given by

$$
S_{\widetilde{V}}(\boldsymbol{\psi})=\frac{1}{|\operatorname{det} \boldsymbol{M}|} \sum_{\boldsymbol{m} \in \mathbb{Z}^{2}} S_{V}\left(\boldsymbol{M}^{-\mathrm{T}}(\boldsymbol{\psi}-2 \pi \boldsymbol{m})\right)
$$

except possibly on a set of Lebesgue measure zero. Hence, the spectral density of the sampled field consists of the superposition of periodically repeated versions of $S_{V}$. Whenever there is some overlap between the supports of these replicas, we speak of aliasing.

The question that remains to be answered is the one about how to reconstruct the analog field $\left\{V(z): z \in \mathbb{R}^{2}\right\}$ from its samples. To start, we rewrite (18) in terms of $\Psi$

$$
S_{\widetilde{V}}\left(\boldsymbol{M}^{\mathrm{T}} \boldsymbol{\Psi}\right)=\frac{1}{|\operatorname{det} \boldsymbol{M}|} \sum_{\boldsymbol{m} \in \mathbb{Z}^{2}} S_{V}\left(\bar{\Psi}-2 \pi \boldsymbol{M}^{-\mathrm{T}} \boldsymbol{m}\right) .
$$

The spectral replicas in (19) are centered at the vertices of the regular lattice generated by the matrix $2 \pi \boldsymbol{M}^{-\mathrm{T}}$, which we denote by $\Lambda_{M}^{*}$ and which we call the spectral lattice. If we denote the support of the spectral density $S_{V}$ by $\mathfrak{S}_{V}$, the support of the function $\boldsymbol{\Psi} \mapsto S_{\widetilde{V}}\left(\boldsymbol{M}^{\mathrm{T}} \boldsymbol{\Psi}\right)$ is given by

$$
\bigcup_{\boldsymbol{k} \in \Lambda_{\boldsymbol{M}}^{*}}\left(\mathfrak{S}_{V}+\boldsymbol{k}\right)
$$

where $\mathfrak{S}_{V}+\boldsymbol{k}$ denotes the set of points of the form $\Psi+\boldsymbol{k}$, for $\Psi \in \mathfrak{S}_{V}$. We say that the lattice $\Lambda_{\boldsymbol{M}}$ allows alias-free sampling of the field $\left\{V(\boldsymbol{z}): z \in \mathbb{R}^{2}\right\}$ if the replicas $\mathfrak{S}_{V}+\boldsymbol{k}$, for $\boldsymbol{k} \in$ $\Lambda_{\boldsymbol{M}}^{*} \backslash\{\boldsymbol{0}\}$, do not overlap with $\mathfrak{S}_{V}$, except possibly on a set of Lebesgue measure zero.

Since the spectral density $S_{V}$ has a compact support, the matrix $\boldsymbol{M}$ can be adjusted so that there is no aliasing. The support of the term corresponding to $\boldsymbol{m}=\mathbf{0}$ in (19), which is $\mathfrak{S}_{V}$, is then contained inside a primitive cell, say $\mathcal{B}$, of the spectral lattice $\Lambda_{M}^{*}$. Hence, we can recover the spectral density $S_{V}$ from $S_{\widetilde{V}}($ knowing $\mathcal{B})$ by writing

$$
S_{V}(\boldsymbol{\Psi})= \begin{cases}|\operatorname{det} \boldsymbol{M}| S_{\widetilde{V}}\left(\boldsymbol{M}^{\mathrm{T}} \boldsymbol{\Psi}\right) & \text { if } \boldsymbol{\Psi} \in \mathcal{B} \\ 0 & \text { otherwise }\end{cases}
$$

i.e., by multiplying $S_{\widetilde{V}}\left(\boldsymbol{M}^{\mathrm{T}} \boldsymbol{\Psi}\right)$ by $\widehat{s}(\boldsymbol{\Psi}):=|\operatorname{det} \boldsymbol{M}| 1_{\mathcal{B}}(\boldsymbol{\Psi})$, where $1_{\mathcal{B}}$ denotes the indicator function of the set $\mathcal{B}$. We therefore choose the inverse Fourier transform of the function $\widehat{s}$ as a candidate for the interpolating function $s: \mathbb{R}^{2} \rightarrow \mathbb{R}$

$$
s(\boldsymbol{z}):=\frac{|\operatorname{det} \boldsymbol{M}|}{(2 \pi)^{2}} \int_{\mathcal{B}} e^{j\langle\boldsymbol{z}, \boldsymbol{\Psi}\rangle} \mathrm{d} \boldsymbol{\Psi} .
$$

Since the spectrum of a homogeneous random field is symmetric with respect to the origin, we always assume, without loss of generality, that the primitive cell $\mathcal{B}$ is symmetric as well. The function $s$ is then indeed a real-valued function. Letting $Q_{L}:=$ $[-L, L]^{2} \cap \mathbb{Z}^{2}$, we define the random fields $\left\{V_{L}(\boldsymbol{z}): \boldsymbol{z} \in \mathbb{R}^{2}\right\}$, for $L \in \mathbb{N}$, as finite linear combinations of the analog field's samples, each sample being weighted by the interpolating function centered at the corresponding vertex of the sampling lattice $\Lambda_{\boldsymbol{M}}$ :

$$
V_{L}(\boldsymbol{z}):=\sum_{\boldsymbol{l} \in Q_{L}} \tilde{V}[\boldsymbol{l}] s(z-\boldsymbol{M l})
$$

Our aim is to prove that for all $z \in \mathbb{R}^{2}$

$$
\lim _{L \rightarrow \infty} \mathrm{E}\left[\left|V(z)-V_{L}(z)\right|^{2}\right]=0 .
$$

For bandlimited stochastic processes on $\mathbb{R}$ and random fields on $\mathbb{R}^{N}$ with bounded spectra of rectangular shape, it is known that convergence in (22) holds for rectangular primitive cells $\mathcal{B}$ satisfying $\mathfrak{S}_{V} \subseteq \mathcal{B}$ (see, for example, [25, Th. 3.7.1], [28], [29]). However, to justify quincunx sampling below, we have to extend these results to more general spectral geometries.

To avoid some potentially pathological cases, we assume that the primitive cell $\mathcal{B}$ can be written as the union of a finite number of convex sets (this mild regularity condition is called $U$-convexity in [24]). Assuming further that $\mathfrak{S}_{V} \subseteq \mathcal{B}$, we then have the following result.

Theorem 1: For each $z \in \mathbb{R}^{2}$,

$$
\lim _{L \rightarrow \infty} \mathrm{E}\left[\left|V(\boldsymbol{z})-V_{L}(\boldsymbol{z})\right|^{2}\right]=0 .
$$

Hence, the analog field $V(x, t)$ can be arbitrarily well approximated by $V_{L}(x, t)$ by taking $L$ sufficiently large.

Proof: Expressing $K_{V}$ in terms of the spectral density $S_{V}$, we obtain

$$
\begin{aligned}
& \mathrm{E}\left[\left|V(\boldsymbol{z})-V_{L}(\boldsymbol{z})\right|^{2}\right]= \\
& \frac{1}{(2 \pi)^{2}} \int_{\mathcal{B}}\left|e^{j\langle\boldsymbol{z}, \boldsymbol{\Psi}\rangle}-\sum_{\boldsymbol{l} \in Q_{L}} e^{j\langle\boldsymbol{M l}, \boldsymbol{\Psi}\rangle} s(\boldsymbol{z}-\boldsymbol{M} \boldsymbol{l})\right|^{2} S_{V}(\Psi) \mathrm{d} \boldsymbol{\Psi},
\end{aligned}
$$

since $S_{V}$ vanishes outside $\mathcal{B}$. Once we show that for each $z \in \mathbb{R}^{2}$

$$
\lim _{L \rightarrow \infty} \sum_{\boldsymbol{l} \in Q_{L}} e^{j\langle\boldsymbol{M l}, \boldsymbol{\Psi}\rangle} s(\boldsymbol{z}-\boldsymbol{M} \boldsymbol{l})=e^{j\langle\boldsymbol{z}, \boldsymbol{\Psi}\rangle}
$$

pointwise in $\Psi \in \mathcal{B}$ (the interior of $\mathcal{B}$ ), the partial sums being uniformly bounded for $\Psi \in \mathcal{B}$ and $L \in \mathbb{N}$, the proposition follows from the dominated convergence theorem and the Lebesgue integrability of the spectral density $S_{V}$. 
For fixed $z \in \mathbb{R}^{2}$, the left-hand side of (23) may be interpreted as the generalized Fourier series representation of the $2 \pi \boldsymbol{M}^{-\mathrm{T}}$-periodic function defined by $\boldsymbol{\Psi} \mapsto e^{j\langle\boldsymbol{z}, \boldsymbol{\Psi}\rangle}$ if $\boldsymbol{\Psi} \in \mathcal{B}$, and extended to $\mathbb{R}^{2}$ by periodicity, the numbers $s(\boldsymbol{z}-\boldsymbol{M l})$ being the Fourier coefficients. As we argue in [24, Sect. 3.4], it follows from the $\cup$-convexity of the primitive cell $\mathcal{B}$ that this function is of bounded harmonic variation [30]. The required convergence in (23) then follows from the main theorem in [31].

For any bandlimited random field $\left\{V(z): z \in \mathbb{R}^{2}\right\}$ we can choose a rectangular spectral lattice with sufficiently large primitive cell $\mathcal{B}$ such that the field's spectrum is contained inside $\mathcal{B}$. However, the area of the primitive cell, $(2 \pi)^{2} /|\operatorname{det} \boldsymbol{M}|$, is directly related to the number of samples per unit area, $1 /|\operatorname{det} \boldsymbol{M}|$. Since increasing the sampling density generally requires the deployment of additional sensors, we aim at choosing $\mathcal{B}$ as small as possible and consider nonrectangular lattices in addition to the rectangular ones. However, the sampling density of any aliasfree sampling lattice $\Lambda_{M}$ satisfies

$$
\frac{1}{|\operatorname{det} \boldsymbol{M}|} \geq \frac{\mu\left(\mathfrak{S}_{V}\right)}{(2 \pi)^{2}}
$$

where $\mu\left(\mathfrak{S}_{V}\right)$ denotes the Lebesgue measure of the random field's spectrum. We say that the lattice $\Lambda_{\boldsymbol{M}}$ allows critical sampling of the random field $V$ if it allows alias-free sampling of $V$ and if there is equality in (24). We therefore call

$$
\rho_{V}:=\frac{\mu\left(\mathfrak{S}_{V}\right)}{(2 \pi)^{2}}
$$

the critical sampling density for the random field $V$. Through a judicious choice of the sampling geometry we may thus reduce the sampling density while still avoiding aliasing. In particular, as we show in what follows, the spectrum of a bandlimited sound field under the far-field assumption cannot be well approximated by rectangles, so that choosing primitive cells of nonrectangular shape is more appropriate.

\section{B. Sampling of the Sound Field}

In the following, we assume, as aforementioned at the end of Section II, that the Fourier transform $\widehat{g}(\Phi, \Omega)$ of the Green's function vanishes whenever $|\Phi|>|\Omega| / c$, and, as mentioned in Section III, that the source field $\left\{U(x, t):(x, t) \in \mathbb{R}^{2}\right\}$ is bandlimited to the wavenumber $\widetilde{\Phi}_{0}$ and to the frequency $\Omega_{0}$, i.e., that its spectral density $S_{U}$ vanishes outside the rectangle $\left(-\widetilde{\Phi}_{0}, \widetilde{\Phi}_{0}\right) \times\left(-\Omega_{0}, \Omega_{0}\right)$. It then follows from (15) that the support of the spectral density $S_{V}$, which is given by the intersection of the supports of $\widehat{g}$ and $S_{U}$, is contained inside the bow-tie shaped region $\mathcal{B}$ illustrated in Fig. 4. Note that the wavenumber bandwidth of the sound field is at most equal to $\Phi_{0}:=\Omega_{0} / c$, independently of the actual wavenumber bandwidth $\widetilde{\Phi}_{0}$ of the source. In what follows, we consider the traditional rectangular sampling lattice as well as the so-called quincunx lattice, which turns out to be particularly well adapted to the spectral characteristics of the sound field. One of the properties of these lattices is that the projection of the vertex set $\left\{\boldsymbol{M l}: \boldsymbol{l} \in \mathbb{Z}^{2}\right\}$ onto the first coordinate forms a regular one-dimensional lattice, so that the deployment of a finite number of sensors per unit distance

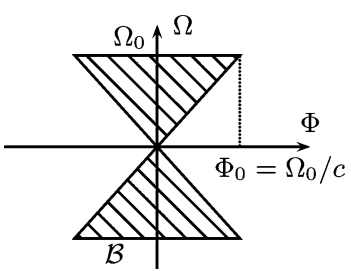

Fig. 4. Bow-tie shaped cell $\mathcal{B}$ containing the support of the sound field's spectral density $S_{V}(\Phi, \Omega)$ in the far-field approximation.

is sufficient, which is an obvious requirement for any practical implementation.

1) Sampling With Rectangular Geometry: The most straightforward generalization of one-dimensional periodic sampling to the two-dimensional case is periodic sampling with rectangular geometry. Since the sound field $\left\{V(x, t):(x, t) \in \mathbb{R}^{2}\right\}$ is bandlimited to the wavenumber $\Omega_{0} / c$ and to the frequency $\Omega_{0}$, it can be sampled without aliasing if the Nyquist condition is satisfied in both the spatial and the temporal dimensions. That is, the sensors are equally spaced on the line $\mathcal{V}$ with an intersensor spacing $X_{0}$ of at most $\pi c / \Omega_{0}$, and each sensor records its measurements with a sampling period $T_{0}$ of at most $\pi / \Omega_{0}$. Furthermore, all the sensors record the samples at the same time instants. Since we do not consider oversampling, we choose $X_{0}:=\pi c / \Omega_{0}$ and $T_{0}:=\pi / \Omega_{0}$. These values define the rectangular sampling lattice $\Lambda_{\boldsymbol{M}_{\mathrm{r}}}$ shown in Fig. 5(a), whose generating matrix may be written as

$$
\boldsymbol{M}_{\mathrm{r}}:=\frac{\pi}{\Omega_{0}}\left(\begin{array}{ll}
c & 0 \\
0 & 1
\end{array}\right) .
$$

The analog field can then be reconstructed from its samples by means of the interpolation with an ideal low-pass filter. The convergence of the corresponding interpolation series is proved in [28] and [29].

As explained in Section IV-A, the spectral density $S_{\widetilde{V}}$ of the sampled field $\left\{\tilde{V}[\boldsymbol{l}]:=V\left(\boldsymbol{M}_{\mathrm{r}} \boldsymbol{l}\right): \boldsymbol{l} \in \mathbb{Z}^{2}\right\}$ is composed of shifted copies of $S_{V}$, and these copies are centered at the vertices of the spectral lattice $\Lambda_{M_{\mathrm{r}}}^{*}$, which is generated by the matrix $2 \pi M_{\mathrm{r}}^{-\mathrm{T}}$. Fig. 5(b) shows that, with our choice of the sampling parameters, the supports of these copies do not overlap, and hence, there is no aliasing. However, the figure also shows that the rectangular sampling lattice is a conservative choice, in the sense that it does not lead to the tightest possible tiling of the wavenumber-frequency plane with the replicas of $S_{V}$. The gaps that remain between the replicas contribute to the correlation of the spatio-temporal samples. Since any spatial processing of the samples is impossible in a distributed setting, a transform coding approach to reduce the correlation, as in [5] and [32], is not an option. Moreover, we show below that the samples' spatial correlation prevents the spatially independent source coding scheme described in Section V-C from leading to a good rate distortion tradeoff. This motivates the choice of a sampling lattice that is better adapted to the spectral characteristics of the sound field.

2) Sampling With Quincunx Geometry: Periodic sampling with quincunx geometry has been suggested in [7] to achieve a tighter packing of the spectral replicas. As mentioned above, 


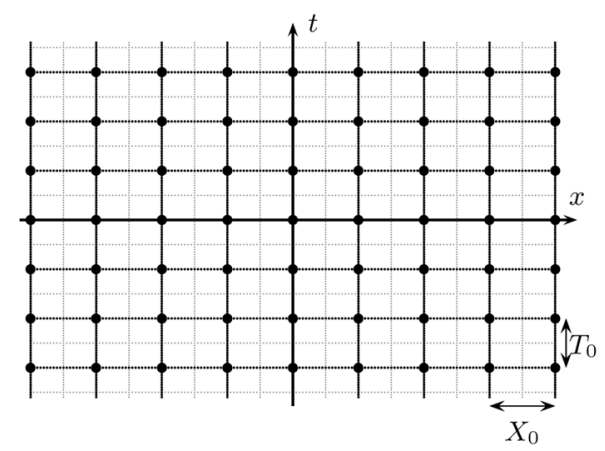

(a)

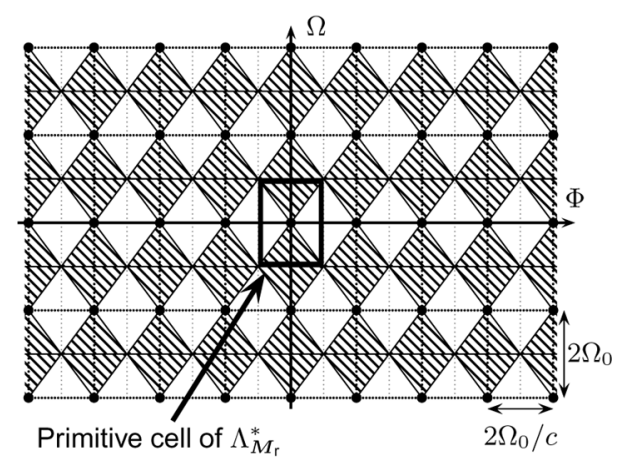

(b)

Fig. 5. Sampling lattice (a) and support of the spectral density of the sampled field (b) for periodic sampling with rectangular geometry. The rectangular lattice allows alias-free sampling, but not critical sampling, of the sound field.

the support of the sound field's spectral density $S_{V}$ is contained inside the bow-tie shaped region $\mathcal{B}$ illustrated in Fig. 4 . The set $\mathcal{B}$ may be regarded as a primitive cell of the rhombic (or centered rectangular) lattice generated by the matrix

$$
\frac{\Omega_{0}}{c}\left(\begin{array}{cc}
1 & 1 \\
-c & c
\end{array}\right) \text {. }
$$

It follows from the discussion in Section IV-A that, if we choose this rhombic lattice as the spectral lattice $\Lambda_{\boldsymbol{M}_{\mathrm{q}}}^{*}$ of our sampling scheme, the shifted copies of the spectral density $S_{V}$ that appear in (18) do not overlap. Hence, the sound field $\{V(x, t)$ : $\left.(x, t) \in \mathbb{R}^{2}\right\}$ can be sampled without aliasing at the vertices of the corresponding sampling lattice $\Lambda_{\boldsymbol{M}_{\mathrm{q}}}$, whose generating matrix may be written as

$$
M_{\mathrm{q}}:=\frac{\pi}{\Omega_{0}}\left(\begin{array}{cc}
c & c \\
-1 & 1
\end{array}\right) .
$$

Although the sampling lattice $\Lambda_{\boldsymbol{M}_{\mathrm{q}}}$, which is shown in Fig. 6(a), is again a rhombic lattice, we call it a quincunx lattice, in keeping with the terminology introduced in [7]. As with the rectangular lattice above, the intersensor spacing is equal to $X_{0}=\pi c / \Omega_{0}$, but each sensor now records its measurements with a sampling period of $2 T_{0}=2 \pi / \Omega_{0}$ and with a temporal offset of $T_{0}$ with respect to its two neighboring sensors. Although each sensor samples the local sound field below the Nyquist rate, the overall sampling density is sufficiently high to avoid aliasing. Even if the quincunx lattice does not allow us to decrease the number of microphones deployed per unit length, it leads to a reduction of the sampling density by a factor of

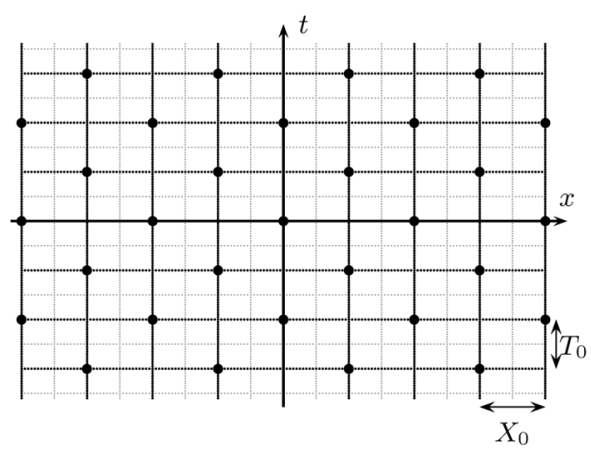

(a)

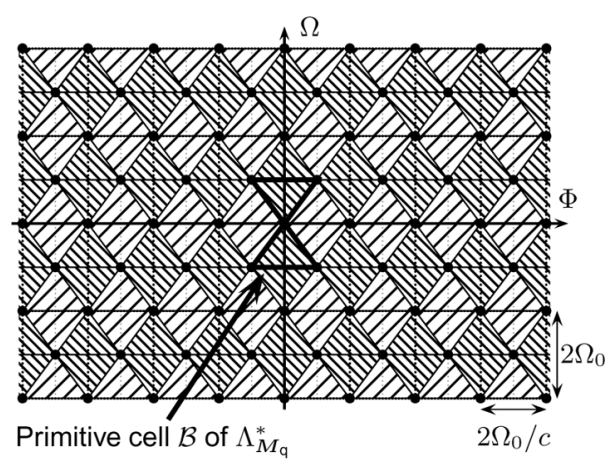

(b)

Fig. 6. Sampling lattice (a) and support of the spectral density of the sampled field (b) for periodic sampling with quincunx geometry. In addition to the replicas obtained with the rectangular lattice, sampling at the vertices of the quincunx lattice generates the light-hatched copies. Thus, the quincunx lattice allows critical sampling of the sound field.

two, as compared with the rectangular lattice. The interpolating function $s_{\mathrm{q}}(x, t)$ to be used with the quincunx lattice is given by (20). Since the primitive cell $\mathcal{B}$ is the union of two convex sets (ter, $\mathcal{B}$ is $\cup$-convex), Theorem 1 allows us to assert that the corresponding interpolation series converges in quadratic mean.

Fig. 6(b) shows that sampling with the quincunx lattice $\Lambda_{\boldsymbol{M}_{\mathrm{q}}}$ leads to a gap-free tiling of the wavenumber-frequency plane with the replicas of $S_{V}$. Hence, the lattice $\Lambda_{M_{\mathrm{q}}}$ allows critical sampling of the sound field $V$. We show below that this property affects the rate distortion tradeoff achieved by the spatially independent source coding scheme in a significant way.

To simplify the arguments in what follows, we perform a change of indices and denote by $\widetilde{V}[k, n]$, for $(k, n) \in \mathbb{Z}^{2}$, the sample $V\left(k X_{0}, t_{k, n}\right)$ taken by the sensor at position $k X_{0}$ and at time

$$
t_{k, n}:=n 2 T_{0}+(k \bmod 2) T_{0} .
$$

\section{SOURCE CODING SCHEMES}

As aforementioned in the Introduction, the sound field $\left\{V(x, t):(x, t) \in \mathbb{R}^{2}\right\}$ along the line $\mathcal{V}$ is sampled by an array of sensors, and its samples $\widetilde{V}[k, n]$ are supposed to be transmitted to the base station through digital channels. That is, each sensor encodes its measurements into a sequence of binary digits, and the base station computes its estimates $\widehat{V}[k, n]$ of the field's samples based on these bits. The setup is shown in 


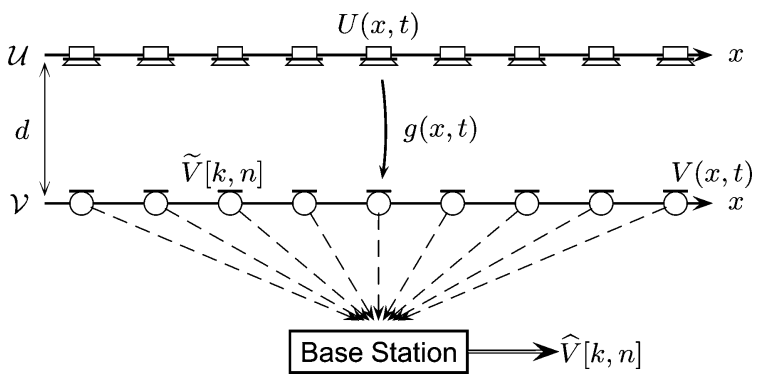

Fig. 7. Sampling and source coding for acoustic wave acquisition. The sensor nodes sample the sound field $V(x, t)$, encode the samples $V[k, n]$ and transmit the encoded samples to the base station through digital channels. The base station computes estimates $\widehat{V}[k, n]$ of the field's samples.

Fig. 7. While in Section IV we have dealt with the sampling operation, we now address the source coding problem.

Since in this paper we address neither the problem of making point-to-point communications reliable nor the one of devising efficient network communication schemes, we assume that the channels are perfect bit pipes carrying the transmitted bits without error to the destination. Furthermore, although joint source-channel coding strategies may potentially lead to an improved performance in general multiterminal networks [1]-[3], our setup corresponds to the "expanding sensor network" model studied in [4] and [5], for which Shannon's (source-channel) separation theorem has been shown to hold, at least in a scaling-law sense, i.e., asymptotically, as the number of sensors grows to infinity. This allows us to address the source coding problem independently of the channel coding problem without sacrificing the optimal performance of the system.

In what follows, we specify the source coding schemes that we let the sensors apply to the samples $\widetilde{V}[k, n]$ before their transmission to the base station. These schemes differ from each other by the amount of explicit collaboration allowed between the sensors and by the extent to which the spatio-temporal correlation of the samples is taken into account. Since we assume that the sensors operate without communicating with each other, the coding scheme that they are supposed to use is multiterminal source coding. However, since we are unable to determine the rate distortion function for this scheme, we consider the centralized and the spatially independent coding schemes. The rate distortion functions that we compute for these schemes provide lower and upper bounds for the multiterminal rate distortion function. While centralized coding achieves the same rate distortion tradeoff for any sampling scheme, provided that there is no aliasing, the performance of spatially independent coding is affected by the chosen sampling lattice, as it depends on how strongly the sound field's samples are correlated in the spatial dimension.

As we assume a digital communication infrastructure, the cost of transmission is measured in terms of the average bit rate, i.e., in terms of the average number of bits per unit length and unit time sent to the base station. As is usual in Gaussian source coding, we choose to measure the quality of the base station's estimates by the mean squared error (MSE) per sample

$$
\frac{1}{2 K+1} \frac{1}{2 N+1} \sum_{|k| \leq K} \sum_{|n| \leq N} \mathrm{E}\left[|\tilde{V}[k, n]-\widehat{V}[k, n]|^{2}\right]
$$

with $K, N \in \mathbb{N}$. The source coding problem can now be formulated as follows: find the minimal average bit rate used by the sensors for a given MSE of the base station's estimates, as $K$, $N \rightarrow \infty$. The optimal tradeoff is typically expressed in terms of a rate distortion function $R(D)$. Furthermore, one can argue, as in [15, Sect. 4.6.3], that this is indeed equivalent to the problem of producing an estimate of the analog field $V(x, t)$ to within a given MSE per unit length and unit time. The choice of the MSE fidelity criterion is consistent with the interpretation of the integral in (13) as a q.m. linear operation as well as with the q.m. convergence of the interpolation series stated in Theorem 1. However, we will argue in Section VI below that, once the sound field has been sampled, the main implication of Theorem 2, namely the one pertaining to the optimality of spatially independent coding, remains valid for any single-letter fidelity criterion. Choosing the MSE measure allows us nevertheless to provide closed-form expressions for the various rate distortion functions. To simplify the notation in the figures below, we let $Z_{k}^{N}$ denote the vector $(Z[k, 0], \ldots, Z[k, N-1])$.

\section{A. Multiterminal Source Coding}

In the multiterminal source coding scheme, both the encoding performed by the sensors and the decoding done at the base station are based on the spatio-temporal correlation of the samples, although the sensors operate without communicating with each other. That is, the encoding is distributed. The setup is shown in Fig. 8. A detailed description of multiterminal source coding is given in [24, Sect. 2.4.1]. This scheme is of highest interest in sensor network engineering as it allows taking the spatial correlation into account without requiring intersensor communication.

It is known that, for discrete-valued, correlated data sources and asymptotically lossless (i.e., zero distortion) compression, the constraint of noncollaborative encoding does not induce any increase in the required rate [33]. However, in the lossy (i.e., nonzero distortion) multiterminal source coding scheme, there is in general a cost in rate due to the separation of the encoders [34]. A complete characterization of the region of achievable rate distortion pairs for that scheme has not been found to date. In her thesis [35], Tung gave inner and outer bounds to this rate distortion region (known today as the Berger-Tung bounds), and Oohama obtained an outer bound for Gaussian sources and MSE distortion that partially coincides with the Berger-Tung inner bound [36]. Zamir and Berger obtained a complete solution for general sources in the extreme of high resolution (i.e., high rate) [34], while Wagner, Tavildar, and Viswanath determined the rate distortion region for the two-terminal Gaussian/MSE problem [37]. (They also provided a partial answer for the setup with several sources, assuming a symmetric covariance structure and symmetric distortion constraints.) It remains unknown how to generalize the latter result to the multiterminal source coding problem with an arbitrary number of sources/terminals, which is the problem of interest in the present paper. That is why we study two other source coding schemes, which provide lower and upper bounds for the multiterminal rate distortion function, namely the centralized and the spatially independent coding schemes. 


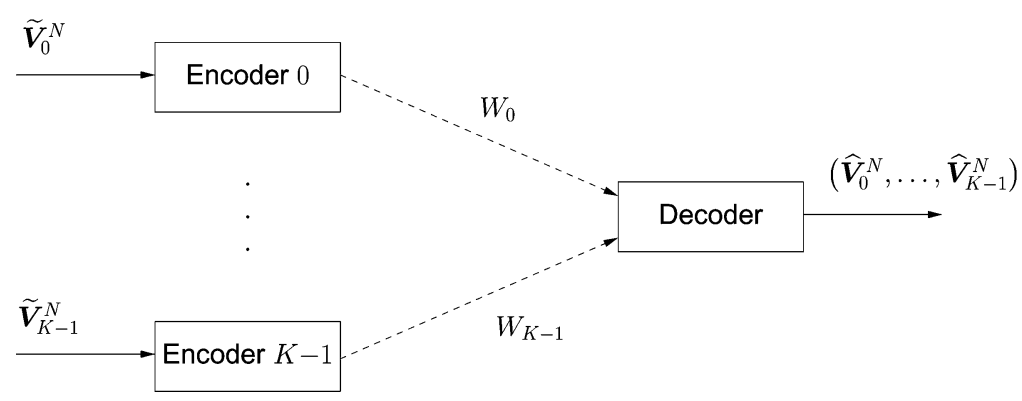

Fig. 8. Multiterminal source coding. The sensors do not communicate while encoding their samples, but the decoding at the base station is performed jointly.

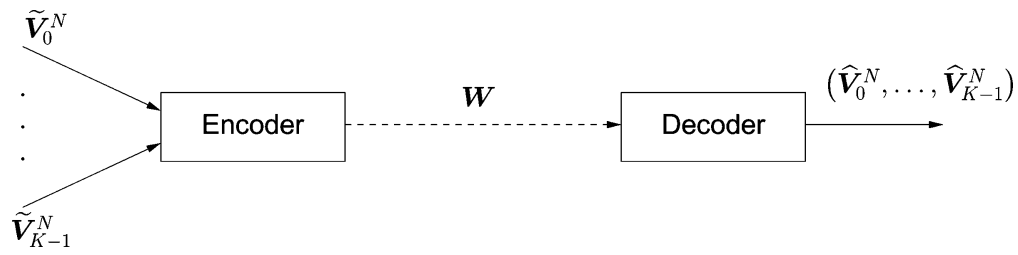

Fig. 9. Centralized source coding. All the spatio-temporal samples are available at a single encoder.
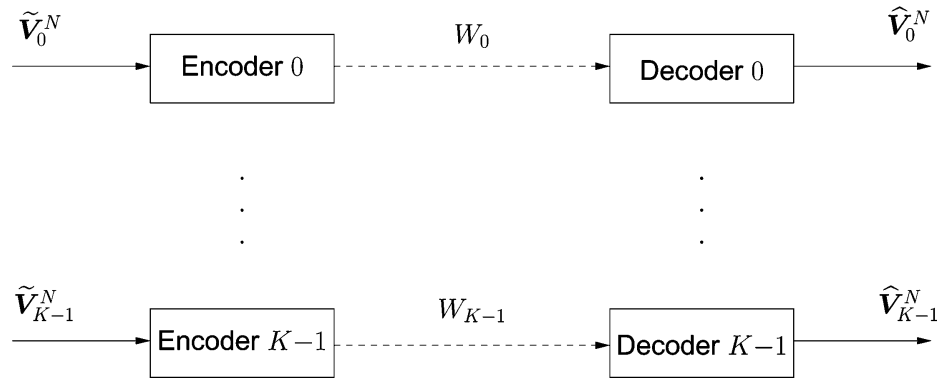

Fig. 10. Spatially independent source coding. Both the encoding at the sensors and the decoding at the base station are performed without cooperation.

\section{B. Centralized Source Coding}

In the centralized source coding scheme, we assume that there is a single encoder that has access to all the spatio-temporal samples of the field $\left\{\widetilde{V}[k, n]:(k, n) \in \mathbb{Z}^{2}\right\}$. We may for instance assume that there is a genie informing each sensor about other sensors' measurements, or, slightly more realistically, that the sensors have unconstrained channels at their disposal to communicate with each other at no cost. As a consequence, the sensors may encode their measurements jointly. The setup is shown in Fig. 9. A detailed description of centralized source coding is given in [24, Sect. 2.4.3].

Using the reverse water-filling principle [15, Corollary 2.8.3] and generalizing [15, Th. 4.5.3] to homogeneous, Gaussian random fields (see also [15, Exercise 4.27]), we obtain the following parametric representation of the rate distortion function for the centralized coding scheme:

$$
\begin{aligned}
& R_{\theta}=\frac{\alpha \Phi_{0} \Omega_{0}}{\pi^{2}} \frac{1}{(2 \pi)^{2}} \\
& \cdot \iint_{(-\pi, \pi]^{2}} \max \left[0, \frac{1}{2} \log \frac{S_{\widetilde{V}}(\phi, \omega)}{\theta}\right] \mathrm{d} \omega \mathrm{d} \phi
\end{aligned}
$$

$$
D_{\theta}=\frac{1}{(2 \pi)^{2}} \iint_{(-\pi, \pi]^{2}} \min \left[\theta, S_{\widetilde{V}}(\phi, \omega)\right] \mathrm{d} \omega \mathrm{d} \phi
$$

where $S_{\widetilde{V}}$ denotes the spectral density of the random field $\left\{\widetilde{V}[k, n]:(k, n) \in \mathbb{Z}^{2}\right\}$, and $\alpha$ is a parameter depending on the chosen sampling lattice. For the rectangular lattice $\alpha=1$, while for the quincunx lattice $\alpha=1 / 2$. The rate has been normalized by the sampling density, so that both the rate and the distortion are computed on a per unit length and unit time basis. Note that, despite the presence of the parameter $\alpha$ in (28), the centralized coding scheme achieves the same rate distortion tradeoff for both the rectangular and the quincunx sampling lattices. Since the centralized source coding scheme allows more flexibility in the design of the encoder and the decoder than the multiterminal coding scheme does, the rate distortion function for centralized coding is a lower bound for the multiterminal rate distortion function.

\section{Spatially Independent Source Coding}

In the spatially independent source coding scheme, we make each sensor encode its measurements ignoring the correlation they have with the samples recorded by the neighboring sensors. Similarly, the base station decodes the bit sequence received from each sensor separately. The setup is shown in Fig. 10. Furthermore, each encoder-decoder pair in Fig. 10 is chosen to implement an efficient point-to-point source code. A detailed description of spatially independent source coding is given in [24, Sect. 2.4.4]. 
The overall performance of the spatially independent coding scheme depends on the characteristics of the stationary, Gaussian random processes that are locally observed by the sensors. By the homogeneity of the sampled sound field $\left\{\widetilde{V}[k, n]:(k, n) \in \mathbb{Z}^{2}\right\}$, these processes all have the same spectral density, which is given by

$$
S_{0}(\omega)=\frac{1}{2 \pi} \int_{(-\pi, \pi]} S_{\widetilde{V}}(\phi, \omega) \mathrm{d} \phi .
$$

The point-to-point rate distortion functions for these processes then follow from [15, Th. 4.5.3]. Regarding the aggregate rate distortion function, the only degree of freedom remaining is the choice of the rate at which each encoder-decoder pair is supposed to operate. By the symmetry of the setup, the homogeneity of the random field, and the convexity of the point-topoint rate distortion functions, we conclude that allocating the same rate to every sensor is optimal. Therefore, we obtain the following parametric representation of the rate distortion function for the spatially independent coding scheme:

$$
\begin{aligned}
& R_{\theta}=\frac{\alpha \Phi_{0} \Omega_{0}}{\pi^{2}} \frac{1}{2 \pi} \int_{(-\pi, \pi]} \max \left[0, \frac{1}{2} \log \frac{S_{0}(\omega)}{\theta}\right] \mathrm{d} \omega \\
& D_{\theta}=\frac{1}{2 \pi} \int_{(-\pi, \pi]} \min \left[\theta, S_{0}(\omega)\right] \mathrm{d} \omega
\end{aligned}
$$

where $\alpha$ is the same lattice-dependent parameter as in Section V-B. As before, both the rate and the distortion are computed on a per unit length and unit time basis. Since the spatially independent source coding scheme imposes stronger constraints on the structure of the encoders and the decoders than the multiterminal coding scheme does, the rate distortion function for spatially independent coding is an upper bound for the multiterminal rate distortion function.

Although the encoding of the samples does not require any communication between the sensors in the present scheme, either the sensors have to cooperate to determine the appropriate sampling time offsets for the quincunx lattice, or these offsets must be configured when the sensors are deployed. In Appendix A, we describe a coding scheme that does not require synchronization and still achieves a tighter packing of the replicas of $S_{V}$ than sampling with rectangular geometry does.

\section{RATE DISTORTION FUNCTIONS}

In this section, we evaluate the rate distortion functions for the various sampling lattices and coding schemes described in Sections IV and V. As mentioned before, we assume that the Fourier transform $\widehat{g}(\Phi, \Omega)$ of the Green's function vanishes whenever $|\Phi|>|\Omega| / c$ (far-field approximation), and that the sound source $\left\{U(x, t):(x, t) \in \mathbb{R}^{2}\right\}$ is a homogeneous, Gaussian random field whose spectral density $S_{U}$ vanishes outside the rectangle $\left(-\widetilde{\Phi}_{0}, \widetilde{\Phi}_{0}\right) \times\left(-\Omega_{0}, \Omega_{0}\right)$. The sound field's spectral density $S_{V}$ follows from (15).

\section{A. Rate Distortion Functions for a White Sound Field}

We first consider the case where the spectral density $S_{V}$ of the sound field is constant on its support, i.e.

$$
S_{V}(\Phi, \Omega)= \begin{cases}\sigma_{V}^{2} & \text { if }(\Phi, \Omega) \in \mathcal{B} \\ 0 & \text { otherwise }\end{cases}
$$

where $\sigma_{V}$ is some positive parameter, and $\mathcal{B}$ denotes the bow-tie shaped region shown in Fig. 4. This setting is a worst-case scenario in the sense that, for given bandwidth and total power, the homogeneous, Gaussian random field that requires the highest bit rate for the centralized source coding scheme is the one whose spectral density is constant on $\mathcal{B}$. Under these assumptions we obtain the following rate distortion functions in closed form:

- centralized coding:

$$
R_{\mathrm{c}}(D)=\frac{\rho_{V}}{2} \log \left(\frac{\sigma_{V}^{2} \rho_{V}}{D}\right)
$$

- sampling with the rectangular lattice (26) followed by spatially independent coding:

$$
\begin{array}{r}
R_{\mathrm{si}, \mathrm{r}}(D)=\rho_{V} \log \left(\frac{\sigma_{V}^{2} \rho_{V}}{e D}\left(1+\sqrt{1-\frac{D}{\sigma_{V}^{2} \rho_{V}}}\right)\right) \\
+\rho_{V}\left(1-\sqrt{1-\frac{D}{\sigma_{V}^{2} \rho_{V}}}\right)
\end{array}
$$

- sampling with the quincunx lattice (27) followed by spatially independent coding:

$$
R_{\mathrm{si}, \mathrm{q}}(D)=\frac{\rho_{V}}{2} \log \left(\frac{\sigma_{V}^{2} \rho_{V}}{D}\right)
$$

where $D \in\left(0, \sigma_{V}^{2} \rho_{V}\right)$, and

$$
\rho_{V}=\frac{\Omega_{0}^{2}}{2 \pi^{2} c}
$$

denotes the critical sampling density for the sound field $V$, as defined by (25). The rate distortion functions above, the derivation of which is given in Appendix B, are plotted in Fig. 11. We have chosen $\sigma_{V}^{2}:=0.0027$ to ensure that the sound field in the present setting has approximately the same total power as the one in Section VI-B with $\sigma_{U}^{2}=1$. Note that (32) and (34) are equal. Hence, in the present setting, the scheme that consists in sampling the sound field with the quincunx lattice, generated by the matrix (27), followed by spatially independent coding achieves the same rate distortion tradeoff as centralized coding. Since this scheme may also be regarded as a multiterminal source coding scheme, and since no multiterminal scheme can outperform centralized coding, the rate distortion function for multiterminal source coding is given by expression (34). This result follows from the independence of the sound field's samples. Indeed, since sampling with the quincunx lattice leads to a gap-free tiling of the wavenumber-frequency plane with the replicas of $S_{V}$, as shown in Fig. 6(b), and since $S_{V}$ is constant on $\mathcal{B}$, the spectral density $S_{\widetilde{V}}$ of the sampled field $\left\{\tilde{V}[k, n]:(k, n) \in \mathbb{Z}^{2}\right\}$ is a constant function. Hence, the random processes sampled by different sensors are independent and may therefore be encoded independently without any loss in terms of the rate distortion tradeoff. Let us summarize the discussion above by the following theorem.

Theorem 2: In the far-field setting and with a bandlimited, white, Gaussian sound field, the rate distortion function for multiterminal source coding is given by

$$
R_{\mathrm{mt}}(D)=\frac{\Omega_{0}^{2}}{4 \pi^{2} c} \log \left(\frac{\sigma_{V}^{2} \Omega_{0}^{2}}{2 \pi^{2} c D}\right) .
$$




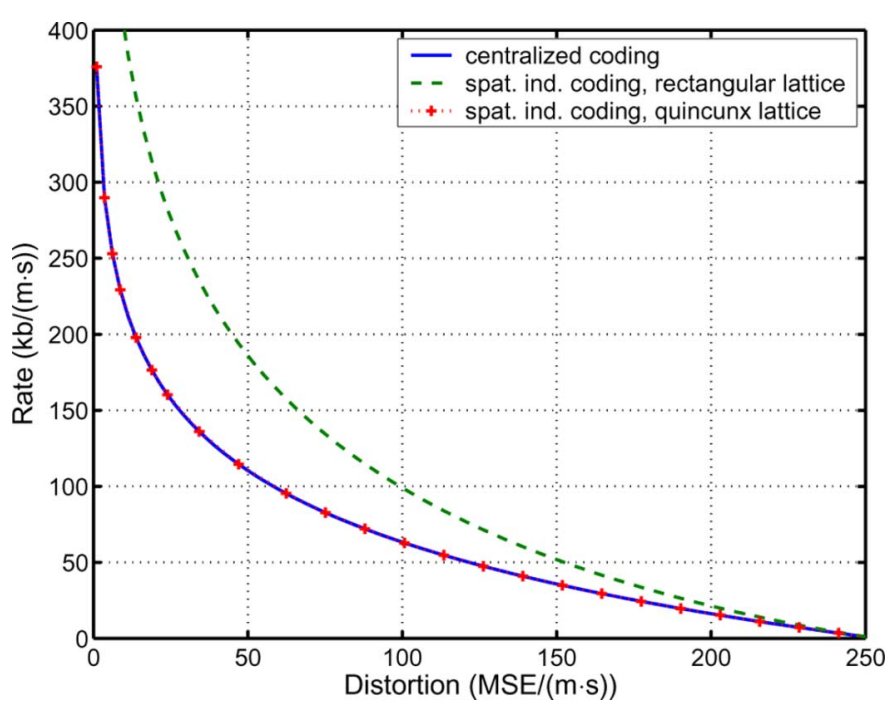

Fig. 11. Rate distortion functions for different sampling lattices and coding schemes in the far-field setting with a bandlimited, white, Gaussian sound field The parameters are $c=340 \mathrm{~m} / \mathrm{s}, \sigma_{V}^{2}=0.0027$, and $\Omega_{0}=8000 \pi \mathrm{rad} / \mathrm{s}$.

Of course, the statement in Theorem 2 is only valid if we choose the parameters of the quincunx sampling lattice so that its primitive cell perfectly matches the support of the sound field's spectral density, and that the gap-free tiling of the wavenumber-frequency plane in Fig. 6(b) is obtained. Indeed, if we choose the sampling density not high enough, aliasing is the consequence, and the sound field cannot be perfectly reconstructed from its samples. However, if the sampling density is too high, the gaps that appear between the shifted copies of $S_{V}$ make the samples correlated and, in general, prevent the spatially independent coding scheme from being optimal.

The proof of Theorem 2 mainly consists in showing that spatially independent coding, preceded by sampling with an appropriately adjusted quincunx lattice, achieves the same rate distortion tradeoff as centralized coding, and this in turn follows from the independence of the sound field's samples. The MSE fidelity criterion, despite allowing us to provide closed-form expressions for the rate distortion functions, plays no role in this regard. With independent samples, independent coding is optimal for any single-letter fidelity criterion of the form

$$
\frac{1}{2 K+1} \frac{1}{2 N+1} \sum_{|k| \leq K} \sum_{|n| \leq N} \mathrm{E}[d(\tilde{V}[k, n], \widehat{V}[k, n])]
$$

where $K, N \in \mathbb{N}$, and $d: \mathbb{R} \times \mathbb{R} \rightarrow[0,+\infty)$ is a measurable distortion function. Hence, we have the following theorem.

Theorem 3: In the far-field setting and with a bandlimited, white, Gaussian sound field, the rate distortion function for multiterminal source coding is equal to those of both centralized and spatially independent coding. This result holds for any singleletter fidelity criterion.

In other words, the judicious choice of the sampling lattice exempts us from having to resort to any multiterminal binning as the same rate distortion tradeoff may be achieved with the computationally less intensive spatially independent source coding scheme.

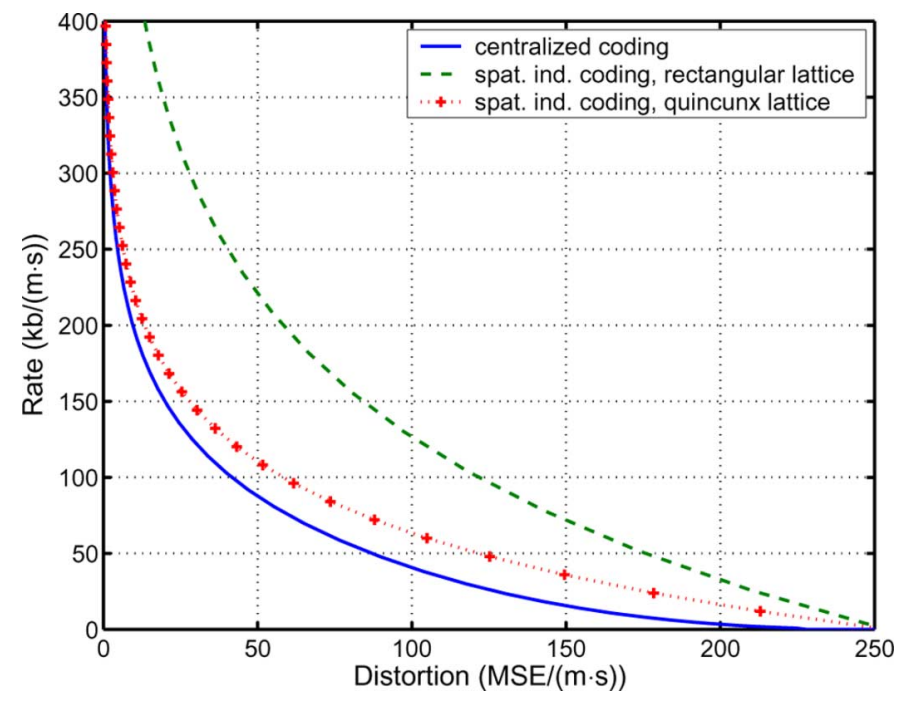

Fig. 12. Rate distortion functions for different sampling lattices and coding schemes in the far-field setting with a bandlimited, white, Gaussian sound source. The parameters are $c=340 \mathrm{~m} / \mathrm{s}, d=10 \mathrm{~m}, \sigma_{U}^{2}=1$, and $\Omega_{0}=8000 \pi \mathrm{rad} / \mathrm{s}$.

\section{B. Rate Distortion Functions for a White Sound Source}

To further study the influence of the wave equation's Green's function on the rate distortion tradeoff, we next consider the case where the source field's spectral density $S_{U}$ is constant on its support, i.e.

$$
S_{U}(\Phi, \Omega)= \begin{cases}\sigma_{U}^{2} & \text { if }(\Phi, \Omega) \in\left(-\widetilde{\Phi}_{0}, \widetilde{\Phi}_{0}\right) \times\left(-\Omega_{0}, \Omega_{0}\right) \\ 0 & \text { otherwise }\end{cases}
$$

where $\sigma_{U}$ is some positive parameter. Then, the sound field's spectral density $S_{V}$, which is given by (15), is no longer constant on the set $\mathcal{B}$. Without loss of generality we assume that $\widetilde{\Phi}_{0}>\Omega_{0} / c$. For this setup we have to resort to numerical integration to compute the various rate distortion functions, which are shown in Fig. 12. Unlike in the previous setting, where $S_{V}$ is constant on $\mathcal{B}$, the spatially independent coding scheme with the quincunx sampling lattice does not achieve the performance of centralized coding, but it still outperforms the scheme with the rectangular sampling lattice by a significant margin. Hence, by adjusting the sampling lattice to the spectral properties of the sound field, we achieve a good performance even with a source coding scheme that does not take into account the spatial correlation of the samples. As a complete characterization of the region of achievable rate distortion pairs for the multiterminal source coding scheme has not been found to date, this scheme's rate distortion function for the present setup remains undetermined within the bounds provided by centralized coding and by spatially independent coding with quincunx sampling.

\section{CONCLUSION}

The problem addressed in this paper is an instance of the multiterminal source coding problem, which has proven to be one of the biggest challenges in information theory. Although some significant progress towards a solution of that problem has been made [37], [38], the question regarding the tightness of the known bounds to the region of achievable rate distortion pairs 


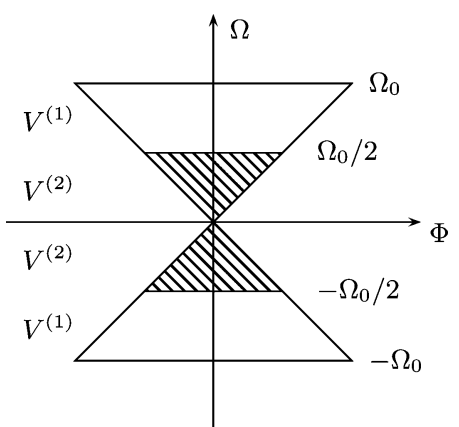

(a)

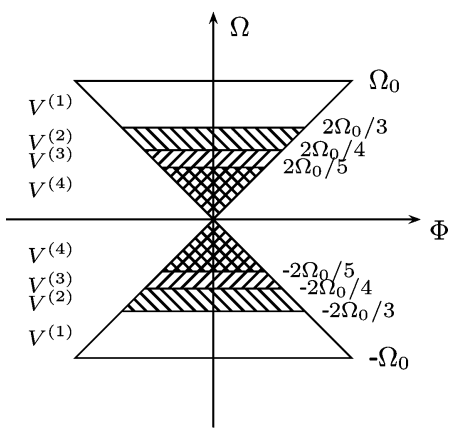

(b)

Fig. 13. Spectral decomposition of the sound field in the subband coding scheme. (a) $L=1, M=2$. (b) $L=2, M=4$.

remains unanswered. The vast majority of studies on multiterminal source coding focuses on Gaussian data sources with arbitrary correlation, without further exploring the physical phenomenon generating the data. While the present paper keeps with the Gaussian/MSE setup, we incorporate both physics and multidimensional sampling into our analysis to obtain a realistic model for the data sources typically encountered in sensor network applications. Based on this model, we determine both upper and lower bounds to the rate distortion function of the multiterminal source coding scheme for the case of acoustic wave acquisition by means of linear sensor arrays. Under the assumption of a worst-case data source, we argue that sampling with a quincunx lattice produces independent samples, exempting us from using multiterminal source coding. Indeed, we show that the rate distortion function of the generally less efficient spatially independent source coding scheme then coincides with the lower bound given by centralized coding, thus establishing the multiterminal rate distortion function for this particular setup. Even though our results do not have immediate implications on the general multiterminal source coding problem, they suggest a guideline for engineering real-world sensor networks and motivate the general advice that communication schemes should be tailored to the physical properties of the underlying problems.

\section{APPENDIX A SubBAND SOURCE CODING}

In what follows, we describe a source coding scheme that, without requiring either intersensor communication or predeployment configuration to set the appropriate sampling time offsets, achieves a tighter packing of the replicas of $S_{V}$ than

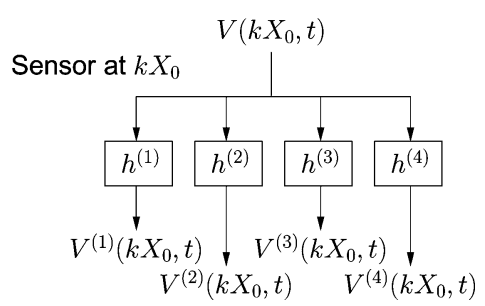

Fig. 14. Filter bank used by the sensors in the subband coding scheme with $M=4$

sampling with rectangular geometry does. The main idea behind this scheme consists in decomposing the sound field into $M$ spectral subbands by means of filtering in the temporal domain, as shown in Fig. 13. Let $\left\{V^{(m)}(x, t):(x, t) \in \mathbb{R}^{2}\right\}$, for $m=1, \ldots, M$, denote the subband fields, which we obtain by making the sensors apply appropriate band-pass filters to the field $\left\{V(x, t):(x, t) \in \mathbb{R}^{2}\right\}$, as shown in Fig. 14. Note that this scheme does not require any spatial filtering, which would preclude its use in any practical setting. Each of the subband fields is sampled at the vertices of a rectangular lattice, whose parameters are adjusted to the wavenumber and the frequency bandwidths of the particular subband field. In general, the implementation of these sampling lattices would require the deployment of a separate set of sensors with the appropriate intersensor spacing for each subband field. To avoid this overhead, we choose the wavenumber bandwidths of the subband fields in such a way that the corresponding spatial Nyquist rates are rational multiples of each other. In that case, we can perform spatial sampling at the Nyquist rate by having a suitable subset of the sensors record the samples of a given subband field. To be explicit, we choose a positive integer $L$ and deploy the sensors with an intersensor spacing of $X_{0} / L=(\pi c) /\left(L \Omega_{0}\right)$, which corresponds to $L$ times the spatial Nyquist rate of the field $\left\{V(x, t):(x, t) \in \mathbb{R}^{2}\right\}$. Then, for each $m=1, \ldots, M$, we define the subband field $\left\{V^{(m)}(x, t):(x, t) \in \mathbb{R}^{2}\right\}$ as the field obtained from the sound field by ideal band-pass filtering with a passband given by

$$
\left\{\begin{array}{l}
{\left[-\frac{L \Omega_{0}}{L+m-1},-\frac{L \Omega_{0}}{L+m}\right] \cup\left[\frac{L \Omega_{0}}{L+m}, \frac{L \Omega_{0}}{L+m-1}\right]} \\
{\left[-\frac{L \Omega_{0}}{L+M-1}, \frac{L \Omega_{0}}{L+M-1}\right] \quad \text { for } m=1, \ldots, M-1}
\end{array}\right.
$$

Fig. 13 shows the resulting decompositions for $L=1$ and $M=2$ [Fig. 13(a)] as well as for $L=2$ and $M=4$ [Fig. 13(b)]. The subband field $V^{(m)}$ has a wavenumber bandwidth of $L \Omega_{0} /(c(L+m-1))$, and thus, it can be sampled with an intersensor spacing of $(L+m-1) X_{0} / L$. We obtain this sampling rate by having one sensor out of $L+m-1$ record the samples of the field $V^{(m)}$

As to source coding, we let the sensors apply the spatially independent coding scheme, defined in Section $\mathrm{V}-\mathrm{C}$, to the samples of each subband field. Since the spectral densities of the subband fields have nonoverlapping supports, these random fields are independent. Hence, they may be encoded independently without any penalty in terms of the rate distortion tradeoff, provided that the rate allocation among the subbands 


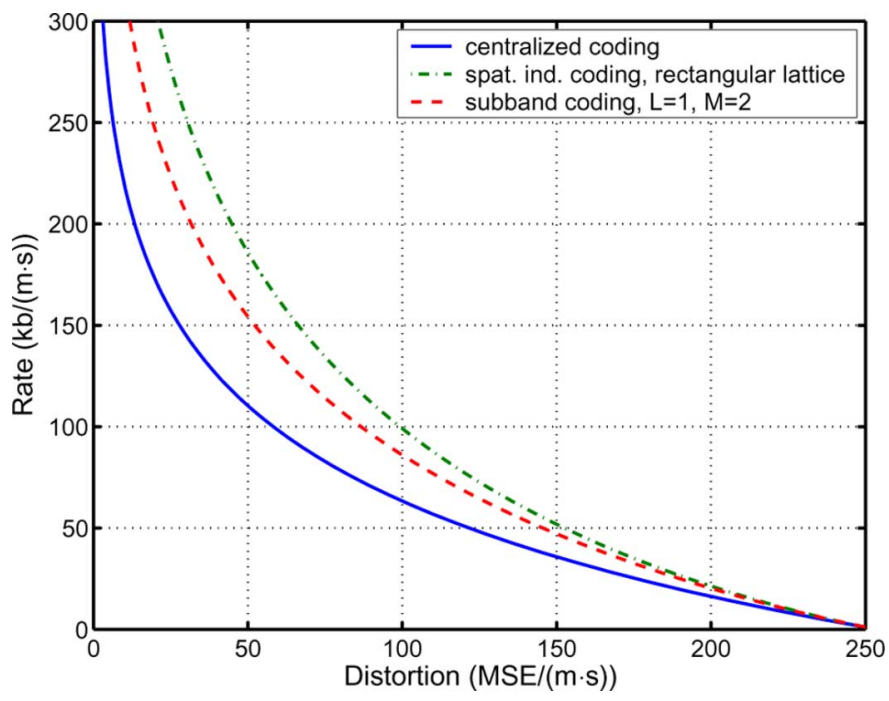

Fig. 15. Rate distortion function for the subband coding scheme with $L=$ 1 and $M=2$, in the far-field setting with a bandlimited, white, Gaussian sound field. The parameters are $c=340 \mathrm{~m} / \mathrm{s}, \sigma_{V}^{2}=0.0027$, and $\Omega_{0}=$ $8000 \pi \mathrm{rad} / \mathrm{s}$.

is performed according to the reverse water-pouring principle [15, Sect. 2.8]. Let $R^{(m)}(D)$, for $m=1, \ldots, M$, denote the rate distortion function for the subband field $V^{(m)}$. The aggregate rate distortion function is then given by

$$
R(D)=\sum_{m=1}^{M} R^{(m)}\left(D^{(m)}\right)
$$

where

$$
D:=\sum_{m=1}^{M} D^{(m)}
$$

and the distortions $D^{(1)}, \ldots, D^{(M)}$ are chosen so that the equalslope condition in [15, Sect. 2.8] is satisfied.

To illustrate the performance of the subband coding scheme defined above, we first consider the decomposition of the sound field into two subbands of equal width, as shown in Fig. 13(a). The sensors are deployed with an intersensor spacing of $X_{0}=\pi c / \Omega_{0}$, and the subband field $V^{(2)}$ is sampled by every other sensor. Fig. 15 shows the resulting rate distortion function in comparison with the ones for centralized coding and for spatially independent coding with the rectangular sampling lattice. Note that, without deploying any additional sensors, we can improve the performance of the spatially independent coding scheme if we combine it with the subband decomposition method. Next, we consider the spectral decomposition into multiple subbands. For a given value of the parameter $L$, we choose $M:=9 L+1$, so that the frequency bandwidth of the field $V^{(M)}$ is equal to $\Omega_{0} / 5$. Note that increasing the parameter $L$ requires a proportional increase of the sensor density, whereas an increase of the number of subbands $M$ only adds to the local processing complexity. Fig. 16 shows the resulting rate distortion functions for $L=1,2,3$. Note that, with a moderate increase of the sensor density, the performance of the spatially independent coding scheme combined with

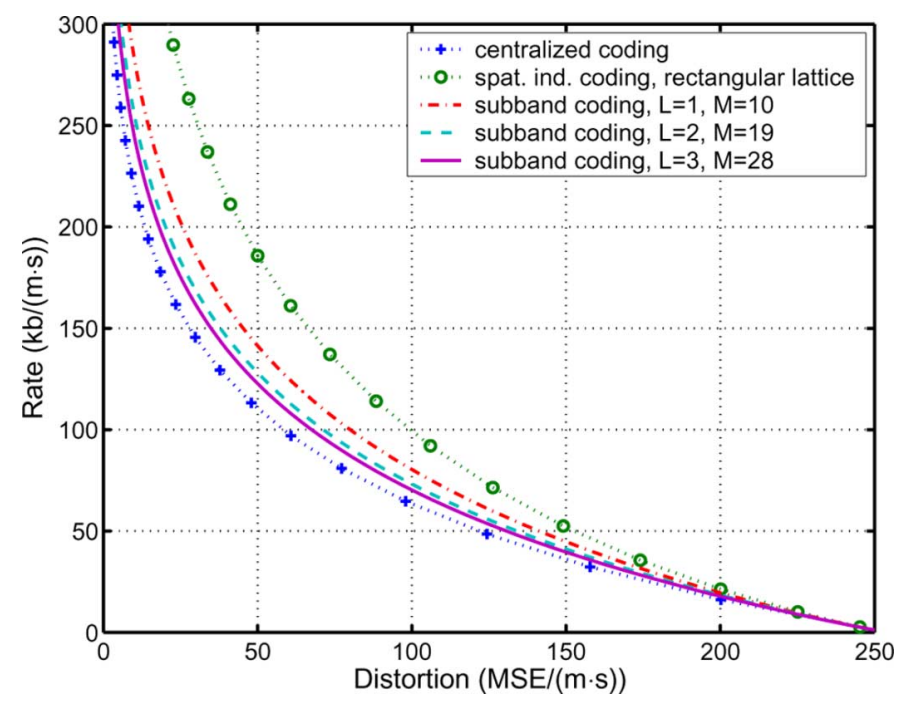

Fig. 16. Rate distortion functions for the subband coding scheme with $L=$ $1,2,3$, and $M=9 L+1$, in the far-field setting with a bandlimited, white, Gaussian sound field. The parameters are $c=340 \mathrm{~m} / \mathrm{s}, \sigma_{V}^{2}=0.0027$, and $\Omega_{0}=8000 \pi \mathrm{rad} / \mathrm{s}$.

the subband decomposition method approaches the one of centralized coding.

\section{APPENDIX B}

\section{RATE Distortion Functions FOR A White SOUND FIELD}

In what follows, we infer the rate distortion tradeoffs (32)-(34) for the acquisition of a bandlimited, white, Gaussian sound field from the general parametric expressions (28)-(31). The spectral density $S_{V}$ of the analog sound field $\left\{V(x, t):(x, t) \in \mathbb{R}^{2}\right\}$ is given by

$$
S_{V}(\Phi, \Omega)=\sigma_{V}^{2} 1_{\mathcal{B}}(\Phi, \Omega)
$$

where $1_{\mathcal{B}}$ denotes the indicator function of the bow-tie shaped region shown in Fig. 4. The critical sampling density $\rho_{V}$ for the field $V$, as defined by (25), is then given by

$$
\rho_{V}=\frac{\Omega_{0}^{2}}{2 \pi^{2} c} .
$$

\section{A. Centralized Coding}

Since the centralized source coding scheme achieves the same rate distortion tradeoff for any sampling lattice, provided that there is no aliasing, we choose the rectangular lattice that is generated by the matrix $\boldsymbol{M}_{\mathrm{r}}$ given by (26). The spectral density $S_{\widetilde{V}}$ : $(-\pi, \pi]^{2} \rightarrow[0,+\infty)$ of the sampled random field $\{\tilde{V}[k, n]:$ $\left.(k, n) \in \mathbb{Z}^{2}\right\}$, which is defined at the end of Section IV-B-1, then follows from (18):

$$
S_{\widetilde{V}}(\phi, \omega)= \begin{cases}\frac{\sigma_{V}^{2} \Omega_{0}^{2}}{\pi^{2} c} & \text { if }|\phi| \leq|\omega| \\ 0 & \text { otherwise. }\end{cases}
$$

Inserting (36) into the parametric expressions (28) and (29), we obtain

$$
R_{\theta}=\frac{\Omega_{0}^{2}}{4 \pi^{2} c} \log \frac{\sigma_{V}^{2} \Omega_{0}^{2}}{\pi^{2} c \theta}
$$




$$
D_{\theta}=\frac{\theta}{2}
$$

for $0<\theta \leq\left(\sigma_{V}^{2} \Omega_{0}^{2}\right) /\left(\pi^{2} c\right)$. Solving for $\theta$ in the latter equation and substituting the result into the former, we get

$$
R_{\theta}=\frac{\Omega_{0}^{2}}{4 \pi^{2} c} \log \frac{\sigma_{V}^{2} \Omega_{0}^{2}}{2 \pi^{2} c D_{\theta}} .
$$

The rate distortion function (32) for centralized coding then follows from (37) and (35).

\section{B. Spatially Independent Coding}

As argued in Section $\mathrm{V}-\mathrm{C}$, the rate distortion function for the spatially independent source coding scheme depends on the spectral density of the stationary, Gaussian random process observed by any one of the sensor nodes. Therefore, to determine this rate distortion tradeoff, we consider the sensor node at position 0 .

1) Rectangular Sampling Lattice: For the rectangular lattice generated by the matrix $\boldsymbol{M}_{\mathrm{r}}$ in (26), it follows from (36) that the spectral density of the sampled random process $\{\widetilde{V}[0, n]$ : $n \in \mathbb{Z}\}$ is given by

$$
\begin{aligned}
S_{\mathrm{r}, 0}(\omega) & =\frac{1}{2 \pi} \int_{(-\pi, \pi]} S_{\widetilde{V}}(\phi, \omega) \mathrm{d} \phi \\
& =\frac{\sigma_{V}^{2} \Omega_{0}^{2}}{\pi^{3} c}|\omega| .
\end{aligned}
$$

Inserting (38) into the parametric expressions (30) and (31), we obtain

$$
\begin{aligned}
R_{\theta} & =\frac{\Omega_{0}^{2}}{\pi^{2} c} \frac{1}{\pi} \int_{\frac{\pi^{3} c \theta}{\sigma_{V}^{2} \Omega_{0}^{2}}}^{\pi} \frac{1}{2} \log \frac{S_{\mathrm{r}, 0}(\omega)}{\theta} \mathrm{d} \omega \\
& =\frac{\Omega_{0}^{2}}{2 \pi^{3} c}\left[\omega \log \frac{\sigma_{V}^{2} \Omega_{0}^{2} \omega}{\pi^{3} c \theta}-\omega\right]_{\omega=\frac{\pi^{3} c \theta}{\sigma_{V}^{2} \Omega_{0}^{2}}}^{\pi} \\
& =\frac{\Omega_{0}^{2}}{2 \pi^{2} c} \log \frac{\sigma_{V}^{2} \Omega_{0}^{2}}{e \pi^{2} c \theta}+\frac{\theta}{2 \sigma_{V}^{2}} \\
D_{\theta} & =\frac{1}{\pi} \int_{0}^{\frac{\pi^{3} c \theta}{\sigma_{V}^{2} \Omega_{0}^{2}}} S_{\mathrm{r}, 0}(\omega) \mathrm{d} \omega+\frac{1}{\pi} \int_{\frac{\pi^{3} c \theta}{\sigma_{V}^{2} \Omega_{0}^{2}}}^{\pi} \theta \mathrm{d} \omega \\
& =\theta-\frac{\pi^{2} c \theta^{2}}{2 \sigma_{V}^{2} \Omega_{0}^{2}},
\end{aligned}
$$

for $0<\theta \leq\left(\sigma_{V}^{2} \Omega_{0}^{2}\right) /\left(\pi^{2} c\right)$. Expressing $\theta$ in terms of $D_{\theta}$, we get

$$
\theta=\frac{\sigma_{V}^{2} \Omega_{0}^{2}}{\pi^{2} c}\left(1-\sqrt{1-\frac{2 \pi^{2} c D_{\theta}}{\sigma_{V}^{2} \Omega_{0}^{2}}}\right) .
$$

Substituting this into the expression for $R_{\theta}$, we obtain

$$
\begin{array}{r}
R_{\theta}=\frac{\Omega_{0}^{2}}{2 \pi^{2} c} \log \left(\frac{\sigma_{V}^{2} \Omega_{0}^{2}}{2 \pi^{2} c e D_{\theta}}\left(1+\sqrt{1-\frac{2 \pi^{2} c D_{\theta}}{\sigma_{V}^{2} \Omega_{0}^{2}}}\right)\right) \\
+\frac{\Omega_{0}^{2}}{2 \pi^{2} c}\left(1-\sqrt{1-\frac{2 \pi^{2} c D_{\theta}}{\sigma_{V}^{2} \Omega_{0}^{2}}}\right)
\end{array}
$$

where we have used the fact that

$$
\left(1-\sqrt{1-\frac{2 \pi^{2} c D_{\theta}}{\sigma_{V}^{2} \Omega_{0}^{2}}}\right)\left(1+\sqrt{1-\frac{2 \pi^{2} c D_{\theta}}{\sigma_{V}^{2} \Omega_{0}^{2}}}\right)=\frac{2 \pi^{2} c D_{\theta}}{\sigma_{V}^{2} \Omega_{0}^{2}} .
$$

The rate distortion function (33) for spatially independent coding preceded by sampling with the rectangular lattice (26) then follows from (39) and (35).

2) Quincunx Sampling Lattice: Since sampling at the vertices of the quincunx lattice that is generated by the matrix $\boldsymbol{M}_{\mathrm{q}}$ given by (27) results in subsampling the random process $\{\widetilde{V}[0, n]: n \in \mathbb{Z}\}$ obtained with the rectangular lattice by a factor 2, the spectral density of the sampled random process observed by the sensor node at position 0 is given by

$$
\begin{aligned}
S_{\mathrm{q}, 0}(\omega) & =\frac{1}{2} S_{\mathrm{r}, 0}(\omega / 2)+\frac{1}{2} S_{\mathrm{r}, 0}(\omega / 2-\pi) \\
& =\frac{\sigma_{V}^{2} \Omega_{0}^{2}}{2 \pi^{2} c} .
\end{aligned}
$$

Inserting (40) into the parametric expressions (30) and (31), we obtain

$$
\begin{aligned}
& R_{\theta}=\frac{\Omega_{0}^{2}}{4 \pi^{2} c} \log \frac{\sigma_{V}^{2} \Omega_{0}^{2}}{2 \pi^{2} c \theta} \\
& D_{\theta}=\theta,
\end{aligned}
$$

for $0<\theta \leq\left(\sigma_{V}^{2} \Omega_{0}^{2}\right) /\left(2 \pi^{2} c\right)$. Substituting the latter equation into the former, we get

$$
R_{\theta}=\frac{\Omega_{0}^{2}}{4 \pi^{2} c} \log \frac{\sigma_{V}^{2} \Omega_{0}^{2}}{2 \pi^{2} c D_{\theta}} .
$$

The rate distortion function (34) for spatially independent coding preceded by sampling with the quincunx lattice (27) then follows from (41) and (35).

\section{REFERENCES}

[1] M. Gastpar, B. Rimoldi, and M. Vetterli, "To code, or not to code: Lossy source-channel communication revisited," IEEE Trans. Inf. Theory, vol. 49, no. 5, pp. 1147-1158, May 2003.

[2] M. Gastpar and M. Vetterli, "Power, spatio-temporal bandwidth, and distortion in large sensor networks," IEEE J. Sel. Areas Commun., vol. 23, no. 4, pp. 745-754, Apr. 2005.

[3] M. Gastpar, "Uncoded transmission is exactly optimal for a simple Gaussian sensor network," IEEE Trans. Inf. Theory, vol. 54, no. 11, pp. 5247-5251, Nov. 2008.

[4] K. Eswaran and M. Gastpar, "On the significance of binning in a scaling-law sense," in Proc. IEEE Information Theory Workshop, Punta del Este, Uruguay, Mar. 2006.

[5] M. Gastpar, P. L. Dragotti, and M. Vetterli, "The distributed Karhunen-Loève transform," IEEE Trans. Inf. Theory, vol. 52, no. 12, pp. 5177-5196, Dec. 2006.

[6] B. Beferull-Lozano and R. L. Konsbruck, "On source coding for distributed temperature sensing with shift-invariant geometries," IEEE Trans. Commun., vol. 59, no. 4, pp. 1053-1065, Apr. 2011.

[7] T. Ajdler, L. Sbaiz, and M. Vetterli, "The plenacoustic function and its sampling," IEEE Trans. Signal Process, vol. 54, no. 10, pp. 3790-3804, Oct. 2006.

[8] E. Telatar, Capacity of Multi-Antenna Gaussian Channels. Florham Park, NJ: AT\&T Bell Labs, Internal Technical Memorandum, 1995.

[9] G. J. Foschini and M. J. Gans, "On limits of wireless communications in a fading environment when using multiple antennas," Wireless Pers. Commun., vol. 6, no. 3, pp. 311-335, Mar. 1998.

[10] A. S. Y. Poon, R. W. Brodersen, and D. N. C. Tse, "Degrees of freedom in multiple-antenna channels: A signal space approach," IEEE Trans. Inf. Theory, vol. 51, no. 2, pp. 523-536, Feb. 2005.

[11] D. Tse and P. Viswanath, Fundamentals of Wireless Communication. Cambridge, U.K.: Cambridge University Press, 2005. 
[12] L. Hanlen and M. Fu, "Wireless communication systems with spatial diversity: A volumetric model," IEEE Trans. Wireless Commun., vol. 5, no. 1, pp. 133-142, Jan. 2006.

[13] M. Franceschetti, M. D. Migliore, and P. Minero, "The capacity of wireless networks: Information-theoretic and physical limits," IEEE Trans. Inf. Theory, vol. 55, no. 8, pp. 3413-3424, Aug. 2009.

[14] D. A. B. Miller, "Communicating with waves between volumes: Evaluating orthogonal spatial channels and limits on coupling strengths," Appl. Opt., vol. 39, no. 11, pp. 1681-1699, Apr. 2000.

[15] T. Berger, Rate Distortion Theory: A Mathematical Basis for Data Compression. Upper Saddle River: Prentice-Hall, 1971.

[16] P. M. Morse and K. U. Ingard, Theoretical Acoustics. New York: McGraw-Hill, 1968.

[17] E. DiBenedetto, Partial Differential Equations. Basel, Switzerland: Birkhäuser, 1995.

[18] T. Ajdler, R. L. Konsbruck, O. Roy, L. Sbaiz, E. Telatar, and M. Vetterli, "Spatio-temporal sampling and distributed compression of the sound field," in Proc. European Signal Processing Conf. (EUSIPCO'06), Florence, Italy, Sep. 2006.

[19] F. Bureau, "Le traité de la lumière de Christian Huygens," Acad. Roy. Belgique. Bull. Cl. Sci., vol. 32, pp. 730-744, Jan. 1946.

[20] A. J. Berkhout, D. de Vries, and P. Vogel, "Acoustic control by wave field synthesis," J. Acoust. Soc. Am., vol. 93, no. 5, pp. 2764-2778, May 1993.

[21] I. S. Gradshteyn and I. M. Ryzhik, Table of Integrals, Series, and Products, 5th ed. New York: Academic, 1996.

[22] P. Billingsley, Probability and Measure, 3rd ed. New York: Wiley, 1995.

[23] J. L. Doob, Stochastic Processes. : John Wiley \& Sons, 1953.

[24] R. L. Konsbruck, "Source Coding in Sensor Networks," Ph.D. thesis, École Polytechnique Fédérale de Lausanne (EPFL), Lausanne, Switzerland, 2009.

[25] E. Wong and B. Hajek, Stochastic Processes in Engineering Systems. New York: Springer-Verlag, 1985.

[26] R. J. Marks, II, Advanced Topics in Shannon Sampling and Interpolation Theory. New York: Springer-Verlag, 1993.

[27] D. E. Dudgeon and R. M. Mersereau, Multidimensional Digital Signal Processing. Upper Saddle River, NJ: Prentice-Hall, 1984.

[28] T. Pogány and P. Peruničić, "On the sampling theorem for homogeneous random fields," Theory Probab. Math. Statist., no. 53, pp. $153-159,1996$.

[29] T. Pogány, "Almost sure sampling restoration of band-limited stochastic signals," in Sampling Theory in Fourier and Signal Analysis: Advanced Topics, J. R. Higgins and R. L. Stens, Eds. Oxford, U.K.: Oxford University Press, 1999.

[30] M. I. Dyachenko and D. Waterman, "Convergence of double Fourier series and $W$-classes," Trans. Amer. Math. Soc., vol. 357, no. 1, pp. 397-407, Jul. 2004.

[31] A. A. Saakyan, "On the convergence of double Fourier series of functions with bounded harmonic variation," Soviet J. Contemp. Math. Anal., vol. 21, no. 6, pp. 1-13, 1986.

[32] V. Goyal, "Theoretical foundations of transform coding," IEEE Signal Process. Mag., vol. 18, no. 5, pp. 9-21, Sep. 2001.

[33] D. Slepian and J. K. Wolf, "Noiseless coding of correlated information sources," IEEE Trans. Inf. Theory, vol. 19, no. 4, pp. 471-480, Jul. 1973.

[34] R. Zamir and T. Berger, "Multiterminal source coding with high resolution," IEEE Trans. Inf. Theory, vol. 45, no. 1, pp. 106-117, Jan. 1999.

[35] S. Y. Tung, "Multiterminal Source Coding," Ph.D. thesis, Cornell Univ., Ithaca, NY, 1978.

[36] Y. Oohama, "Gaussian multiterminal source coding," IEEE Trans. Inf. Theory, vol. 43, no. 6, pp. 1912-1923, Nov. 1997.

[37] A. B. Wagner, S. Tavildar, and P. Viswanath, "Rate region of the quadratic Gaussian two-encoder source-coding problem," IEEE Trans. Inf. Theory, vol. 54, no. 5, pp. 1938-1961, May 2008.

[38] A. B. Wagner and V. Anantharam, "An improved outer bound for multiterminal source coding," IEEE Trans. Inf. Theory, vol. 54, no. 5, pp. 1919-1937, May 2008.

Robert L. Konsbruck (S'06-M'10) received the Dipl.Ing. degree in communication systems from the École Polytechnique Fédérale de Lausanne (EPFL),
Switzerland, in 2002, and the M.Sc. degree in mathematics from Université de Fribourg, Switzerland, in 2007. He received the Ph.D. degree in computer and communication sciences from EPFL in 2009, where he was working along with Prof. M. Vetterli and Prof. E. Telatar on information theoretic questions related to sensor networks used for environmental monitoring.

He has been teaching mathematics at the Lycée Classique d'Echternach, Luxembourg, and is an Adjunct Senior Lecturer at the University of Luxembourg. $\mathrm{He}$ is currently with the Inspection Générale de la Sécurité Sociale, Luxembourg, where he is working on stochastic models for life tables. His research focuses on stochastic modeling of physical phenomena, on evaluating the impact of the laws of physics on the source coding performance of sensor networks, as well as on multidimensional sampling of random fields.

Dr. Konsbruck was awarded the Jean Landry Prize (2002) from EPFL for his diploma thesis and the Prix Jeunes Chercheurs (2007) from the Swiss Academy of Sciences for his Master's thesis.

Emre Telatar (S'88-M'91-SM'11-F'12) received the B.Sc. degree in electrical engineering from the Middle East Technical University, Ankara, Turkey, in 1986 and the S.M. and Ph.D. degrees in electrical engineering and computer science from the Massachusetts Institute of Technology, Cambridge, in 1988 and 1992, respectively.

From 1992 to 1999, he was with the Mathematical Sciences Research Center, AT\&T Bell Laboratories, Murray Hill, NJ. Since 1999, he has been a Professor at the École Polytechnique Fédérale de Lausanne (EPFL), Switzerland. His research interests are in communication and information theories.

Dr. Telatar was the recipient of the IEEE Information Theory Society Paper Award in 2001. He was a Program Co-Chair for the IEEE International Symposium on Information Theory in 2002, and an Associate Editor for Shannon Theory for the IEEE TRANSACTIONS ON INFORMATION THEORY from 2001 to 2004. He was awarded the EPFL Agepoly teaching prize in 2005.

Martin Vetterli (S'86-M'90-SM'91-F'95) received the Dipl. El.-Ing. degree from ETHZ, Zurich, Switzerland, in 1981, the M.Sc. degree from Stanford University, CA, in 1982, and the Doctorat ès Sciences degree from the École Polytechnique Fédérale de Lausanne (EPFL), Switzerland, in 1986.

$\mathrm{He}$ was a Research Assistant at Stanford University and EPFL, and has worked for Siemens and AT\&T Bell Laboratories. In 1986, he joined Columbia University, New York, where he was an Associate Professor of Electrical Engineering and co-director of the Image and Advanced Television Laboratory. In 1993, he joined the University of California, Berkeley, where he was a Professor with the Department of Electrical Engineering and Computer Sciences until 1997, and now holds an Adjunct Professor position. Since 1995, he has been a Professor of Communication Systems at EPFL, where he chaired the Communications Systems Division (1996/1997), and heads the Audiovisual Communications Laboratory. From 2001 to 2004, he directed the National Center of Competence in Research on Mobile Information and Communication Systems. He has also been a Vice-President at EPFL since October 2004 in charge of, among others, international affairs and computing services. He has held visiting positions at ETHZ (1990) and Stanford (1998). He has published about 150 journal papers on a variety of topics in signal/image processing and communications, coauthored three books, and holds a dozen patents. His research interests include sampling, wavelets, multirate signal processing, computational complexity, signal processing for communications, digital image/video processing, joint source/channel coding, signal processing for sensor networks, and inverse problems like acoustic tomography.

Dr. Vetterli is a fellow of ACM, a fellow of EURASIP, and a member of SIAM. He received the Best Paper Award of EURASIP in 1984, the Research Prize of the Brown Bovery Corporation (Switzerland) in 1986, the IEEE Signal Processing Society's Senior Awards in 1991, in 1996 and in 2006 (for papers with D. LeGall, K. Ramchandran, and P. Marziliano, and T. Blu, respectively). He won the Swiss National Latsis Prize in 1996, the SPIE Presidential Award in 1999, the IEEE Signal Processing Technical Achievement Award in 2001, and the IEEE Signal Processing Society Award in 2010. He was a member of the Swiss Council on Science and Technology until December 2003. 